\title{
RHOBTB3 promotes proteasomal degradation of HIF $\alpha$ through facilitating hydroxylation and suppresses the Warburg effect
}

Chen-Song Zhang ${ }^{1, *}$, Qi Liu ${ }^{1, *}$, Mengqi $\mathrm{Li}^{1,{ }^{*},}$, Shu-Yong Lin ${ }^{1, *}$, Yongying Peng ${ }^{1}$, Di Wu ${ }^{1}$, Terytty Yang $\mathrm{Li}^{1}$, Qiang $\mathrm{Fu}^{2}$, Weiping $\mathrm{Jia}^{3}$, Xinjun $\mathrm{Wang}^{4}$, Teng $\mathrm{Ma}^{1}$, Yue Zong ${ }^{1}$, Jiwen Cui ${ }^{1}$, Chengfei Pu${ }^{1}$, Guili Lian ${ }^{1}$, Huiling Guo ${ }^{1}$, Zhiyun $\mathrm{Ye}^{1}$, Sheng-Cai Lin ${ }^{1}$

${ }^{I}$ State Key Laboratory of Cellular Stress Biology, Innovation Center for Cell Signaling Network, School of Life Sciences, Xiamen University, Xiamen, Fujian 361005, China; ${ }^{2}$ Department of Urology, Shanghai Jiao Tong University Affiliated Sixth People's Hospital, Shanghai 200233, China; ${ }^{3}$ Department of Endocrinology and Metabolism, Shanghai Jiao Tong University Affiliated Sixth People's Hospital, Shanghai 200233, China; ${ }^{4}$ Department of Urology, Zhongshan Hospital, Xiamen University, Xiamen, Fujian 361004, China

Hypoxia-inducible factors (HIFs) are master regulators of adaptive responses to low oxygen, and their $\alpha$-subunits are rapidly degraded through the ubiquitination-dependent proteasomal pathway after hydroxylation. Aberrant accumulation or activation of HIFs is closely linked to many types of cancer. However, how hydroxylation of HIF $\alpha$ and its delivery to the ubiquitination machinery are regulated remains unclear. Here we show that Rho-related BTB domain-containing protein 3 (RHOBTB3) directly interacts with the hydroxylase PHD2 to promote HIF $\alpha$ hydroxylation. RHOBTB3 also directly interacts with the von Hippel-Lindau (VHL) protein, a component of the E3 ubiquitin ligase complex, facilitating ubiquitination of HIF $\alpha$. Remarkably, RHOBTB3 dimerizes with LIMD1, and constructs a RHOBTB3/LIMD1-PHD2-VHL-HIF $\alpha$ complex to effect the maximal degradation of HIF $\alpha$. Hypoxia reduces the RHOBTB3-centered complex formation, resulting in an accumulation of HIF $\alpha$. Importantly, the expression level of RHOBTB3 is greatly reduced in human renal carcinomas, and RHOBTB3 deficiency significantly elevates the Warburg effect and accelerates xenograft growth. Our work thus reveals that RHOBTB3 serves as a scaffold to organize a multi-subunit complex that promotes the hydroxylation, ubiquitination and degradation of HIF $\alpha$.

Keywords: RHOBTB3; HIF; PHD; VHL; hydroxylation; ubiquitination; the Warburg effect

Cell Research (2015) 25:1025-1042. doi:10.1038/cr.2015.90; published online 28 July 2015

\section{Introduction}

Oxygen is an essential element for the growth and survival of aerobic organisms. Metazoans have evolved sophisticated molecular mechanisms that sense insufficient supply of oxygen and induce adaptive physiological changes, including metabolic reprogramming, to maintain balance of bioenergetics and to eradicate excessive

*These four authors contributed equally to this work. Correspondence: Sheng-Cai Lin

Tel: +86 592218 2993; Fax: +86 5922182993

E-mail: linsc@xmu.edu.cn

Received 6 April 2015; revised 22 May 2015; accepted 11 June 2015; published online 28 July 2015 free radicals. Inadequate oxygen supply, or hypoxia, is a common feature of solid tumors. In order to sustain continuous growth and proliferation, tumor cells also modify their metabolism to adapt to challenging hypoxic environments [1-3].

The hypoxia-inducible factors (HIFs) are master mediators of the cellular adaptive response to hypoxia $[1,2$, $4,5]$. HIFs are obligate heterodimers consisting of an oxygen-labile $\alpha$-subunit and a stable $\beta$-subunit (ARNT) [6, 7]. Mammals express three isoforms of HIF $\alpha$, of which HIF $1 \alpha$ and HIF $2 \alpha$ (also known as EPAS1) are best characterized $[3,8,9]$. HIFs recognize and bind to hypoxia response elements (HREs) of target genes related to many aspects of cancer growth, including proliferation, angiogenesis and metastasis [7, 10-17]. Notably, many 
of the HIF-upregulated genes also encode enzymes in metabolic pathways $[14,18,19]$. These include glucose transporters such as glucose transporter type 1 (GLUT1) and glycolytic enzymes such as hexokinase-2 (HK2) and lactate dehydrogenase a chain (LDHA), which promote glycolysis and lactate fermentation, respectively [2023]. HIFs also upregulate the expression of pyruvate dehydrogenase kinase 1 (PDK1), which phosphorylates and inhibits the activity of pyruvate dehydrogenase, thus repressing the flux of pyruvate into the TCA cycle [2426]. Exaggerated expression of HIFs therefore can result in a shift towards the Warburg effect characterized by a high rate of glycolysis [27-30]. In addition, abnormally stabilized HIF $1 \alpha$ directs glucose-mediated ribose synthesis preferentially through the non-oxidative pentose phosphate pathway [31]. Not surprisingly, solid tumors often exhibit high levels of HIF $\alpha$ and increased HIF $\alpha$ expression correlates with poor clinical prognosis in many cancer types [4, 10, 32, 33]. Overall, HIFs reprogram glucose metabolism from oxidative to anaerobic ATP production, lower the $\mathrm{O}_{2}$ consumption [34], decrease the ROS level $[35,36]$ and suppress cancer cell death [37, 38].

The protein levels of HIF $\alpha$ isoforms are strictly regulated. Under normoxia, $\mathrm{HIF} \alpha$ proteins are rapidly degraded $[7,39]$. The degradation of HIF $\alpha$ requires hydroxylation and subsequent ubiquitination of the proteins before they are targeted to the proteasomes. Hydroxylation of HIF $\alpha$ occurs on the conserved proline residues within the oxygen-dependent degradation (ODD) domain $[40,41]$, and is catalyzed by the dioxygenase prolyl hydroxylases (PHDs, also known as egg-laying-defective nine family members or EGLNs) [42]. Higher metazoans contain three PHD paralogs (PHD1-PHD3), among them PHD2 appears to be the primary HIF $\alpha$ hydroxylase [4346]. Under normoxic condition, high intracellular oxygen tension directly stimulates PHD2-mediated hydroxylation $[1,45]$. Hydroxylation of HIF $\alpha$ can also be promoted or inhibited by various co-factors [47-49]; however, the regulatory mechanism is still poorly defined. The hydroxylation renders HIF $\alpha$ an increased affinity for the von Hippel-Lindau (VHL) protein, a tumor suppressor frequently mutated in a variety of cancers such as renal carcinoma [50-52]. As a component of ubiquitin E3 ligase complex, VHL mediates HIF $\alpha$ polyubiquitination for proteasomal degradation $[2,53,54]$. Chronic hypoxia or sustained activation of HIF signaling in turn promotes the expression of PHD2, which limits accumulation of HIF $1 \alpha$ in the prolonged exposure to hypoxia and leads to accelerated degradation of HIF $1 \alpha$ upon reoxygenation [55-57]. Importantly, it was previously shown that the LIM domain-containing protein 1 (LIMD1) reduces the stability of HIF $\alpha$ by promoting VHL-mediated ubiquitination of HIF $\alpha$ [58]. In addition, knockdown of LIMD1 promotes tumor growth, and it was thus proposed as a tumor suppressor $[59,60]$. However, how cells regulate HIF $\alpha$ signaling in normoxic and hypoxic conditions by coordinating the hydroxylation, ubiquitination and proteasomal degradation of HIF $\alpha$ remains unclear. Furthermore, recent reports have suggested a lysosome-dependent pathway as an alternative mechanism for $\mathrm{HIF} \alpha$ degradation [61-63].

Rho-related BTB domain-containing protein 3 (RHOBTB3), along with RHOBTB1 and RHOBTB2, is an atypical member of the RHO family. RHOBTB3 differs substantially from the other two members $(\sim 48 \%$ identity) [64] and was shown to function as a regulator controlling protein transport from endosome to the Golgi network. RHOBTB3 was also reported to be a component of CULLIN3 (CUL3)-dependent E3 ubiquitin ligase complex, which is responsible for the degradation of cyclin E and MUF-1 [65-67]. Unlike most of the other members of RHO family being small GTPases, RHOBTB3 is an ATPase and the ATPase activity is critical for its function [68]. Here we show that RHOBTB3 has an essential role in controlling the dynamic stability of HIF $\alpha$. Mechanistically, RHOBTB3 can simultaneously interact with PHD2 and VHL, and these interactions stimulate PHD2's hydroxylase activity and facilitate the ubiquitination of HIF $\alpha$. RHOBTB3 is able to form homodimers or interact with LIMD1 to form a heterodimer, with the latter being favored and more potent in interacting with PHD2 and VHL. Consistently, cells deficient in both RHOBTB3 and LIMD1 have higher levels of HIF $\alpha$ than cells lacking either protein alone. Intriguingly, the interaction between RHOBTB3 and HIF $\alpha$-VHL-PHD2 is weakened in hypoxic condition, allowing for adaptive $\mathrm{HIF} \alpha$ accumulation during hypoxia. In addition, we show that deficiency of RHOBTB3 promotes the Warburg effect. Furthermore, loss of RHOBTB3 significantly accelerates the growth of tumors in xenograft models. Collectively, our study identifies RHOBTB3 as a novel scaffolding protein for a multi-subunit complex that promotes HIF $\alpha$ degradation under both normoxic and hypoxic conditions, thereby suppressing the Warburg effect and preventing tumorigenesis.

\section{Results}

\section{RHOBTB3 downregulates HIF $\alpha$ protein levels}

In an effort to study the regulation of HIF $\alpha$, we identified RHOBTB3 as an interacting protein for VHL in a yeast two-hybrid screen (Supplementary information, Figure S1A). To explore the functional linkage, we first 
generated MEF cells from $R H O B T B 3^{-/-}$mice, and found that RHOBTB3 null cells had significantly elevated levels of all three HIF $\alpha$ isoforms (HIF $1 \alpha-3 \alpha$ ) under normoxic and hypoxic conditions and after cobalt chloride $\left(\mathrm{CoCl}_{2}\right)$ treatment compared with WT MEFs (Figure 1A and Supplementary information, Figure S1B and S1C), suggesting that RHOBTB3 exerts a negative effect on $\mathrm{HIF} \alpha$ expression. Transcriptional activities of HIF, measured in an HRE-luciferase reporter activity assay, were also significantly increased in the RHOBTB3-null MEFs (Supplementary information, Figure S1D). Consistently, reintroducing RHOBTB3 into $\mathrm{RHOBTB}^{-/-} \mathrm{MEFs}$
A

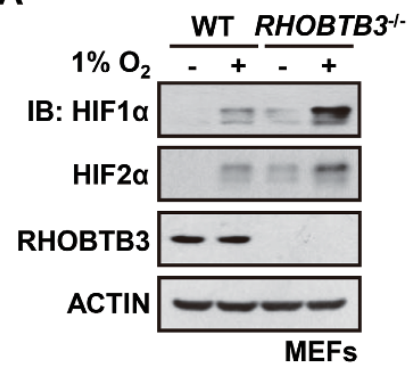

B
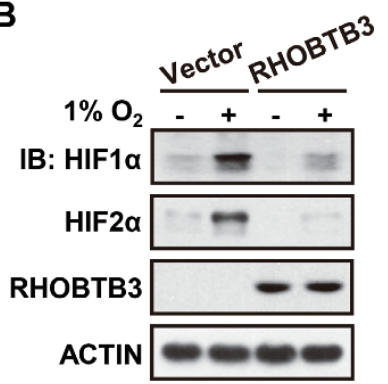

RHOBTB3 $^{-1-}$ MEFs

E

MYC-RHOBTB3 $\quad 0 \quad 1.5 \quad 3 \quad(\mu \mathrm{g})$

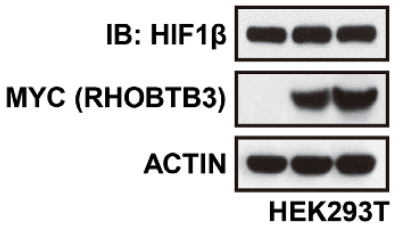

C

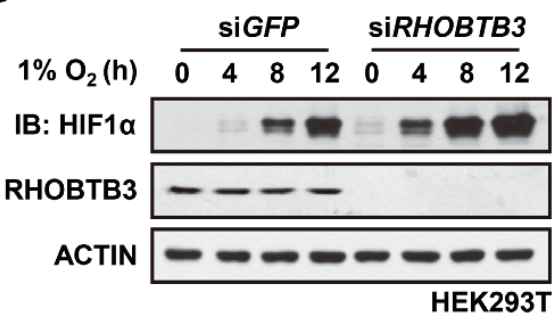

D

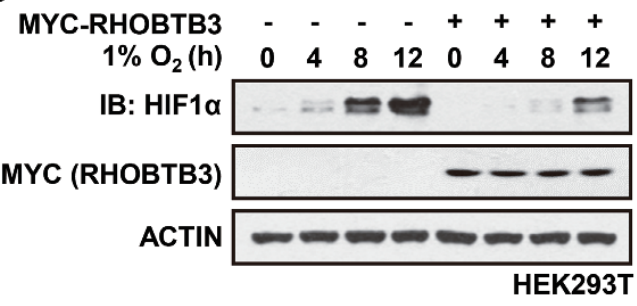

$\mathbf{F}$

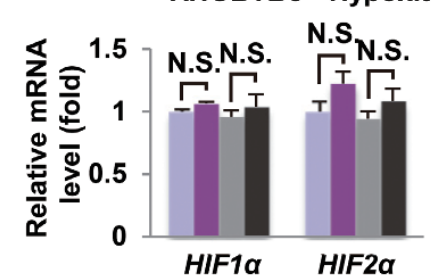

Figure 1 RHOBTB3 downregulates HIF1 $\alpha$ expression. (A) Protein levels of HIF1 $\alpha$ and HIF2 $\alpha$ are elevated in RHOBTB3 ${ }^{-1-}$ MEFs under both normoxic and hypoxic conditions. RHOBTB3 ${ }^{-/}$MEFs and control (WT) MEFs were maintained in normoxia or exposed to hypoxia $\left(1 \% \mathrm{O}_{2}\right)$ for $8 \mathrm{~h}$. Cells were then lysed and analyzed by immunoblotting with antibodies indicated. (B)

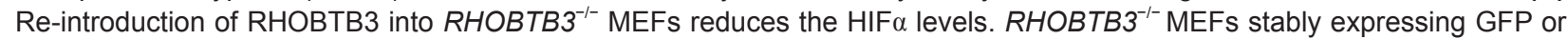
RHOBTB3 were maintained in normoxia or exposed to hypoxia for $8 \mathrm{~h}$, and were then lysed and analyzed as described in $\mathbf{A}$. (C) Knockdown of RHOBTB3 increases the protein levels of HIF1 $\alpha$. HEK293T cells were infected with lentiviruses expressing siRNA targeting either GFP (control) or RHOBTB3. At $16 \mathrm{~h}$ post-infection, cells were exposed to hypoxia for different periods of time as indicated, and were then lysed and analyzed by immunoblotting with antibodies indicated. (D) Ectopic expression of RHOBTB3 in HEK293T cells downregulates HIF1 $\alpha$. HEK293T cells were transfected with pcDNA3.3-MYC-RHOBTB3 or pcDNA3.3-MYC vector as a control. At $16 \mathrm{~h}$ post-transfection, cells were exposed to hypoxia for the indicated periods of time and were then lysed, and the protein levels of HIF1 $\alpha$ were analyzed. (E) RHOBTB3 does not affect the protein levels of HIF1B/ARNT. HEK293T cells were transfected with RHOBTB3. At $16 \mathrm{~h}$ post-transfection, cells were lysed and analyzed by immunoblotting with antibodies indicated. (F) RHOBTB3 has no effect on the mRNA levels of HIF1 $\alpha$ or HIF2 $\alpha$ under both

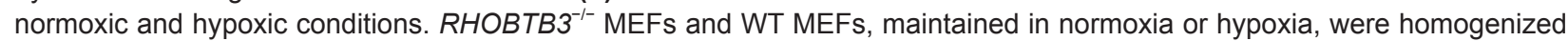
in Trizol reagent, and total RNAs were purified, and were subjected to real-time PCR analysis for mRNA levels of HIF1 $\alpha$ and $H I F 2 \alpha$. Values are presented as mean \pm SEM; $n=3$ for each group; three replicate experiments. N.S., not significant. Statistical analysis was carried out by ANOVA followed by Tukey. 
restored the inhibition on both protein levels and transcriptional activities of HIF $\alpha$ (Figure 1B and Supplementary information, Figure S1D). We also knocked down RHOBTB3 in HEK293T cells, and detected an increase of HIF $1 \alpha$ at the protein levels (Figure 1C). Conversely, ectopic expression of RHOBTB3 in HEK293T cells strongly reduced the protein levels and transcriptional activity of HIF1 $\alpha$ (Figure 1D and Supplementary information, Figure S1E). Unlike HIF1 $\alpha$, the protein levels of HIF1ß/ARNT were not changed by RHOBTB3 overexpression in HEK293T cells (Figure 1E). Compared with RHOBTB3, overexpression of RHOBTB1 or RHOBTB2 had no effect on the protein levels of HIF $1 \alpha$ in HEK293T cells (Supplementary information, Figure S1F). Of note, there was no difference in the mRNA levels of $H I F 1 \alpha$ and HIF $2 \alpha$ between WT and RHOBTB3 ${ }^{-1-}$ MEFs under both normoxic and hypoxic conditions (Figure 1F), indicating that RHOBTB3 selectively affects the protein levels of HIF $\alpha$. Overall, these results suggest that RHOBTB3 strongly downregulates the basal protein levels of HIF $\alpha$ and its induction under hypoxia.

RHOBTB 3 promotes HIF $\alpha$ hydroxylation and ubiquitination in a PHD2- and VHL-dependent manner

We then explored the mechanism by which RHOBTB3 downregulates the protein levels of HIF $\alpha$. In the presence of lysosomal inhibitor chloroquine, RHOBTB3 could still suppress the protein levels of HIF $1 \alpha$, while addition of MG-132 strongly blocked RHOBTB3-mediated HIF $1 \alpha$ degradation, suggesting that RHOBTB3 promotes HIF $1 \alpha$ degradation in a proteasome-specific manner (Supplementary information, Figure S2A). We next explored the possibility that RHOBTB3 promotes HIF $\alpha$ hydroxylation and ubiquitination, two essential modifications prior to proteasomal degradation. While the total protein levels of HIF $1 \alpha$ and its target gene, PHD1-3 was increased in $R H O B T B 3^{-/-}$MEFs, the level of its hydroxylation at proline-564 (OH-P564) was significantly reduced under normoxic or hypoxic conditions (Figure 2A and Supplementary information, Figure S2B), indicating that RHOBTB3 is important for the hydroxylation of HIF $1 \alpha$. Consistently, overexpression of RHOBTB3 effectively downregulated wild-type HIF $1 \alpha$, but had little effect on the hydroxylation-defective mutant P564A (Supplementary information, Figure S2C). To confirm the role of RHOBTB3 in HIF1 $\alpha$ hydroxylation, we carried out in vitro hydroxylation assays. We mixed bacterially expressed ODD domain of HIF1 $\alpha$ (aa 401-603 of human HIF $1 \alpha$ ) with different cell lysates, and found that lysate from RHOBTB3-overexpressing cells strongly stimulated the hydroxylation of HIF1 $\alpha$ (OH-P564) (Figure 2B). Conversely, hydroxylation at P564 of HIF $1 \alpha$ was significantly reduced when the cell lysate prepared from $R H O B T B 3^{-/-}$MEFs was added, even though higher protein levels of PHD2 hydroxylase were present in these cells, and the level of OH-P564 was increased by the lysate from $R H O B T B 3^{-/}$MEFs supplemented with RHOBTB3 (Figure 2B). Moreover, adding in vitro translated RHOBTB3 protein into the lysates of $R H O B T B 3^{-/-}$ MEFs markedly enhanced the OH-P564 levels of HIF1 $\alpha$ (Figure 2C), excluding the possibility that RHOBTB3 regulates hydroxylation through affecting cellular concentrations of other factors such as co-factors for PHDs $[46,69]$. Depletion of PHD2 strongly impaired the RHOBTB3-induced hydroxylation and degradation of HIF $1 \alpha$ in HEK293T cells (Supplementary information, Figure S2D and S2E). Moreover, double knockdown of RHOBTB 3 and PHD2 did not significantly increase the protein levels or transcriptional activity of HIF $1 \alpha$ compared with PHD2 single knockdown (Figure 2D and 2E), suggesting that RHOBTB3 and PHD2 function in the same pathway.

It is known that hydroxylated HIF $1 \alpha$ exhibits increased affinity for VHL $[51,52,70]$. We thus carried out a co-immunoprecipitation experiment to determine whether the interaction between HIF $1 \alpha$ and VHL could be affected by RHOBTB3. Indeed, the interaction was increased in HEK293T cells overexpressing RHOBTB3 under both normoxic and hypoxic conditions (Figure $2 \mathrm{~F}$ ), indicating that more HIF $\alpha$ is recruited to VHL in the presence of RHOBTB3. As a control, RHOBTB3 did not enhance the interaction between the mutant of HIF $1 \alpha$ lacking hydroxylated proline residues (HIF $1 \alpha$ P402AP564A) and VHL (Supplementary information, Figure S2F). Furthermore, in vitro translated RHOBTB3 did not enhance the interaction between bacterially expressed ODD domain (aa 401-603) of HIF1 $\alpha$ (hydroxylation free) and VHL (Supplementary information, Figure S2G). These results strongly indicate that RHOBTB3 enhances HIF $1 \alpha$-VHL interaction by promoting hydroxylation. Elevated levels of ubiquitinated HIF $1 \alpha$ were observed in RHOBTB3-overexpressing HEK293T cells (Supplementary information, Figure S2H). Furthermore, knockdown of PHD2 strongly dampened HIF1 $\alpha$ ubiquitination enhanced by RHOBTB3 (Figure 2G). Moreover, overexpression or knockdown of RHOBTB3 had little effect on the protein levels of HIF $1 \alpha$ in $V H L$ knocked down HEK293 cells (Figure 2H and Supplementary information, Figure S2I). We also found that RHOBTB3 promoted the hydroxylation and ubiquitination of HIF $2 \alpha$ (Supplementary information, Figure S2J). Together, these results suggest that RHOBTB3 downregulates the protein levels of HIF $\alpha$ through promoting hydroxylation and ubiquitination of HIF $\alpha$, which depends on PHD2 and 
A

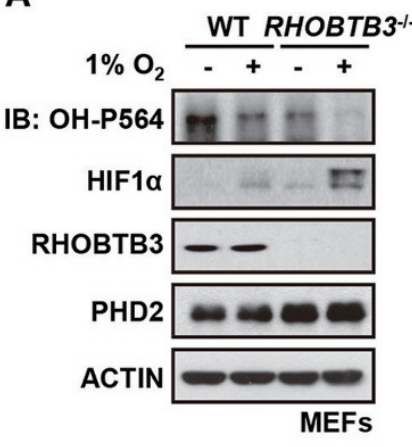

C

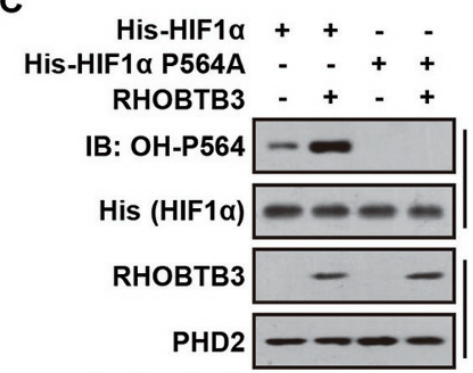

In vitro hydroxylation assay

E

$$
\begin{aligned}
& \text { " siGFP } \\
& \text { = siRHOBTB3 } \\
& \text { = siPHD2 } \\
& \text { - siRHOBTB3\&PHD2 }
\end{aligned}
$$

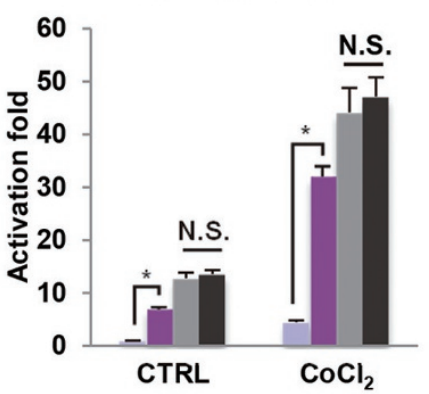

G

$$
\begin{aligned}
& \text { MYC-HIF1a } \frac{\text { SIGFP }}{++} \frac{\text { SiPHD2 }}{++} \\
& \text { HA-RHOBTB3 - }+-+ \\
& \text { FLAG-UB }++++ \\
& \text { MG-132 + + + + }
\end{aligned}
$$$$
\text { IB: FLAG (UB) }
$$$$
\text { MN }
$$

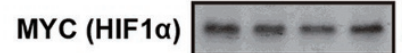$$
\text { IP: }
$$$$
\text { MYC }
$$$$
\text { HA (RHOBTB3) }
$$$$
\text { MYC (HIF1 } \alpha)
$$

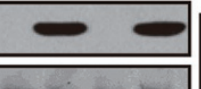$$
\text { PHD2 }
$$

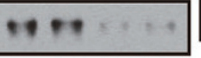

B

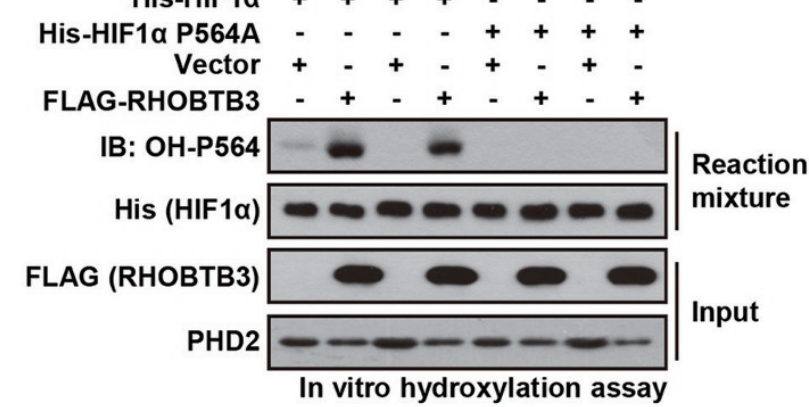

D

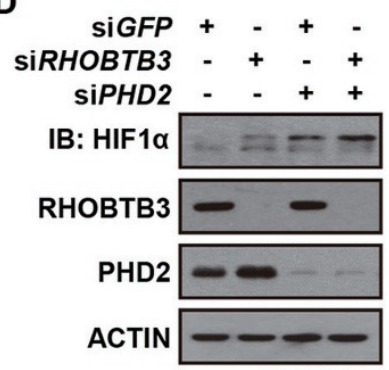

HEK293T

$\mathbf{F}$

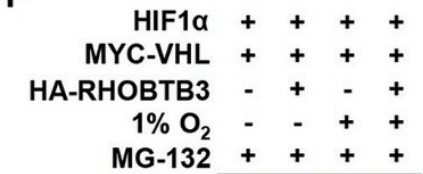

IB: HIF1a

MYC (VHL)

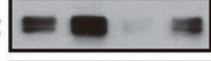

IP: MYC

HIF1 $\alpha$

MYC (VHL)

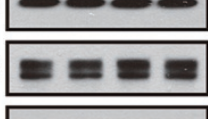

HA (RHOBTB3)

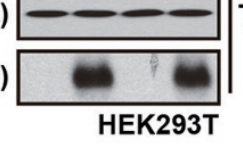
TCL

H

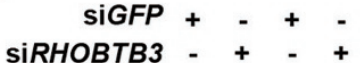

$$
\begin{aligned}
& \text { siVHL - - + + } \\
& \text { IB: HIF1 } \alpha
\end{aligned}
$$

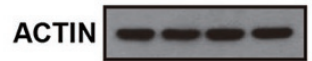


VHL, respectively.

RHOBTB3, PHD2 and VHL form a complex

We next asked whether RHOBTB3 forms a complex with VHL and PHD2, particularly since RHOBTB3 and VHL showed interaction in our yeast two-hybrid screen (Supplementary information, Figure S1A) and that RHOBTB3 promoted PHD2-mediated HIF $\alpha$ hydroxylation. We found that endogenous or ectopically expressed RHOBTB3 was co-immunoprecipitated with VHL or vice versa, but not with the control IgG (Figure 3A and Supplementary information, Figure S3A). Consistently, immunofluorescent staining revealed the co-localization of RHOBTB3 with VHL (Supplementary information, Figure S3B). Similarly, PHD2 was readily co-precipitated with RHOBTB3 (Figure 3A and Supplementary information, Figure S3C). We next asked whether RHOBTB3, PHD2 and VHL co-exist in the same complex. It was observed that RHOBTB3 interacted simultaneously with PHD2 and VHL (Figure 3B). We further employed a two-step co-immunoprecipitation (IP) assay, and found that all three components were detected in the final immunoprecipitates (Figure 3C), indicating their co-existence in the same complex. Of note, HSP90, reported as a chaperone of HIFs [71, 72] and PHD2 [73], was co-precipitated with RHOBTB3 only when HIF1 $\alpha$ was present, suggesting that the HSP90 does not directly interact with RHOBTB3 (Supplementary information, Figure S3D). Although HSP90 has been shown to interact with PHD2 [73] and RHOBTB3 interacts with PHD2 as shown in the present study, PHD2 could co-precipitate HSP90 and RHOBTB3 only when HIF $1 \alpha$ was present, suggesting the PHD2-HSP90 complex depends on HIF $1 \alpha$ to form a co-complex with RHOBTB3 (Supplementary information, Figure S3D). In the presence of ectopic HIF1 $\alpha$, more HSP90 was co-precipitated with RHOBTB3 under normoxia (Supplementary information, Figure S3E), consistent with a stronger interaction of HIF $1 \alpha$ with RHOBTB3 under this condition (Figure 2F). These data indicate that HSP90 does not directly interact with RHOBTB3.

Domain mapping using a series of deletion mutants

Figure 2 RHOBTB3 promotes HIF $\alpha$ hydroxylation and ubiquitination in a PHD2- and VHL-dependent manner. (A) RHOBTB3 promotes hydroxylation of HIF1 $\alpha$ in MEFs. RHOBTB3 ${ }^{-/-}$MEFs and WT MEFs were maintained in normoxia or exposed to hypoxia for $8 \mathrm{~h}$. Cells were then lysed and the hydroxylation on proline-564 of HIF1 $\alpha$ (OH-P564) was analyzed by immunoblotting. As a consequence of sustained accumulation of HIF1 $\alpha$ in RHOBTB3-null MEFs, the protein levels of PHD2 were increased. In contrast, the relatively short-term, 8-h hypoxic exposure did not change the protein levels of PHD2. (B) Ectopically expressed RHOBTB3 promotes hydroxylation of HIF1 $\alpha$ in vitro. RHOBTB3 ${ }^{-1-}$ MEFs and WT MEFs were infected with blank lentiviruses or lentiviruses expressing FLAG-RHOBTB3. Following lysis, the cell lysates were incubated with nickel affinity resin-bound bacterially expressed His-HIF1 $\alpha$ (aa 401-603) or the P564A mutant for 90 min at $30{ }^{\circ} \mathrm{C}$. The mixtures were diluted twofold in a $2 \times$ SDS buffer, and analyzed by western blotting using antibodies indicated. (C) In vitro translated RHOBTB3 promotes hydroxylation of HIF1 $\alpha$. In vitro translated RHOBTB3 and His-HIF1 $\alpha$ (aa 401-603) or the P564A mutant were separately added to cell lysates of $\mathrm{RHOBTB}^{-1-} \mathrm{MEFs}$, and the mixtures were incubated at $30{ }^{\circ} \mathrm{C}$ for 90 min. The mixtures were then analyzed for levels of HIF1 $\alpha$ hydroxylation as in B. (D) Knockdown of PHD2 impairs RHOBTB3-induced degradation of HIF1 $\alpha$. HEK293T cells were infected by lentiviruses expressing control siRNA (GFP), or siRNA targeting RHOBTB3 or PHD2 or both. At $16 \mathrm{~h}$ post-infection, cells were exposed to hypoxia for $4 \mathrm{~h}$, then lysed and analyzed by immunoblotting with antibodies indicated. (E) Double knockdown of RHOBTB3 and PHD2 does not significantly increase transcriptional activity of HIF1 $\alpha$ in single knockdown of PHD2. HEK293T cells were infected with lentiviruses expressing different siRNAs as indicated. After $12 \mathrm{~h}$, cells were treated with $200 \mu \mathrm{M} \mathrm{CoCl}$ for another $8 \mathrm{~h}$ and then lysed. The firefly luciferase reporter carrying HRE was measured and normalized against the Renilla luciferase activity in a dual luciferase assay system. Data are presented as mean \pm SEM; $n=3$ for each group; ${ }^{*} P<0.05$ (ANOVA followed by Tukey); N.S., not significant. (F) RHOBTB3 promotes the interaction between HIF1 $\alpha$ and VHL. HEK293T cells were transfected with different combinations of HIF1 $\alpha$, MYC-VHL and HA-RHOBTB3. At $16 \mathrm{~h}$ post-transfection, cells were treated with $10 \mu \mathrm{M}$ MG-132 and maintained in normoxia or exposed to hypoxia for another $10 \mathrm{~h}$, and were lysed. The protein extracts were immunoprecipitated with antibody against MYC for VHL, and were subjected to western blot analysis. TCL, total cell lysate. (G) Knockdown of PHD2 attenuates RHOBTB3-induced ubiquitination of HIF1 $\alpha$. HEK293T cells infected with lentiviruses expressing siRNA targeting GFP (control) or PHD2 were transfected with different combinations of MYC-HIF1 $\alpha$, HA-RHOBTB3 and FLAG-UB. At $16 \mathrm{~h}$ post-transfection, cells were treated with $10 \mu \mathrm{M}$ MG-132 for another $10 \mathrm{~h}$, and were then lysed with RIPA buffer containing $1 \%$ SDS and boiled. The protein extracts were diluted in RIPA buffer without SDS to a final concentration of $0.2 \%$ SDS, and were subjected to IP with antibody against MYC for HIF1 $\alpha$. The IP product was analyzed by immunoblotting. (H) Knockdown of VHL impairs RHOBTB3 deficiency-induced HIF1 $\alpha$ accumulation. HEK293T cells were infected by lentiviruses expressing siRNA targeting GFP (control), RHOBTB3 and/or VHL. At $16 \mathrm{~h}$ post-infection, cells were exposed to hypoxia for $4 \mathrm{~h}$, and analyzed by immunoblotting to determine HIF1 $\alpha$ protein levels. Probably owing to the low expression levels of its $24 \mathrm{kDa}$ isoform in HEK293T as described previously [80] and the preferential affinity of antibody, only the $19 \mathrm{kDa}$ isoform of VHL (VHL (p19)) could be detected and is shown here. 
A

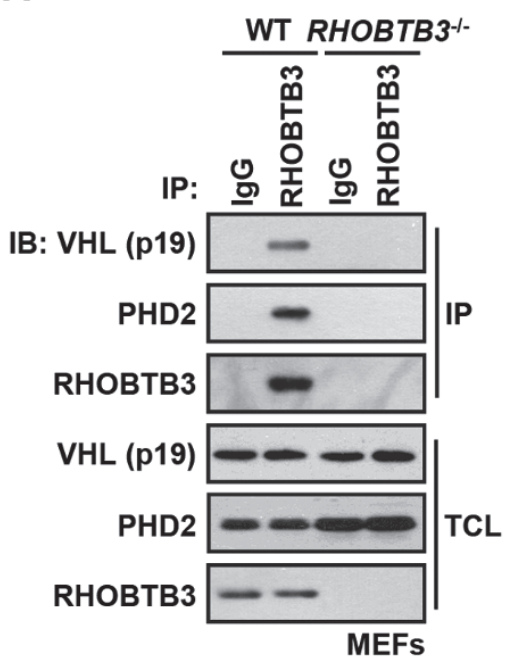

C
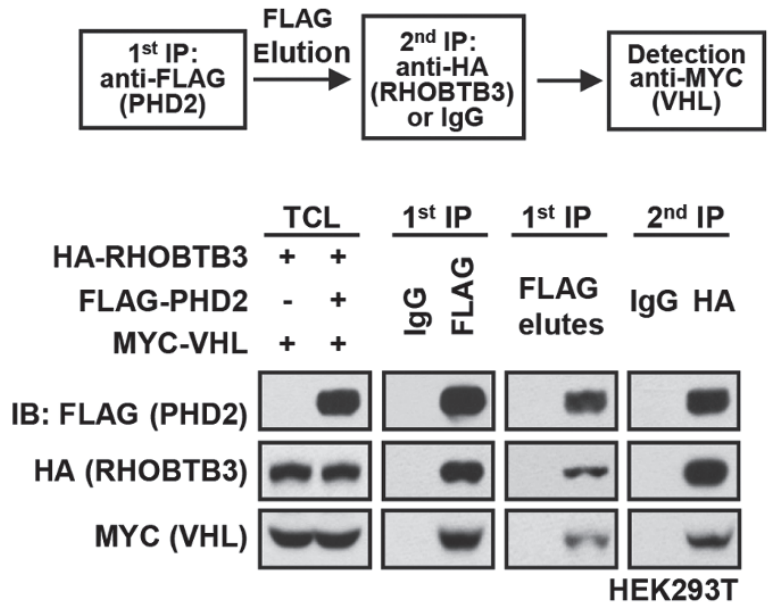

B

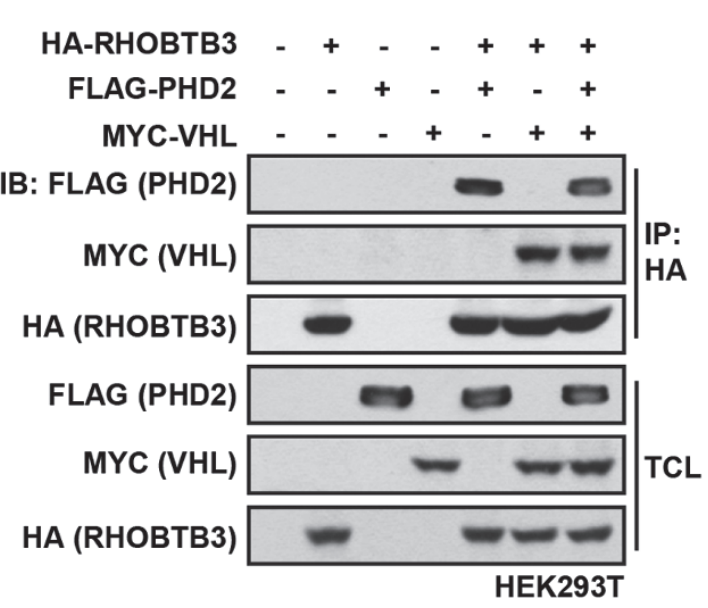

E

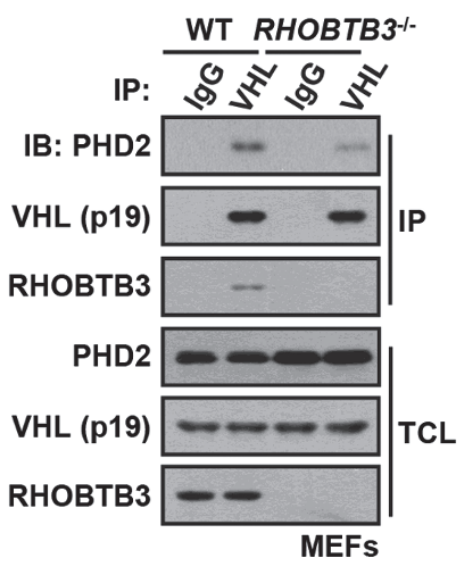

IB: GST (PHD2)
D

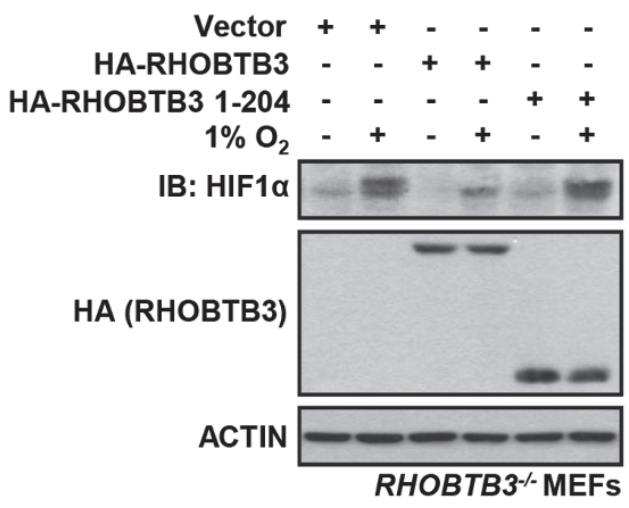

G

\begin{tabular}{|c|c|c|}
\hline HIF1a & + & \\
\hline FLAG-PHD2 & + & + \\
\hline MYC-VHL & + & + \\
\hline HA-RHOBTB3 & + & \\
\hline $\begin{array}{r}1 \% O_{2} \\
M G-132\end{array}$ & $\begin{array}{l}- \\
+\end{array}$ & \\
\hline
\end{tabular}

MYC-VHL - - + - + +

RHOBTB3 - - - + - +

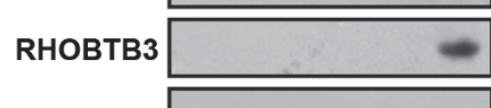

Pull

FLAG (PHD2)

$-\ldots$

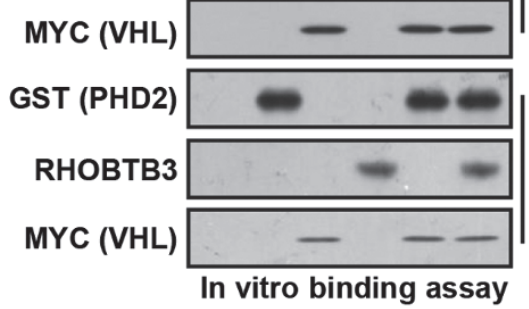

down: HA (RHOBTB3) MYC

IP: MYC

TCL

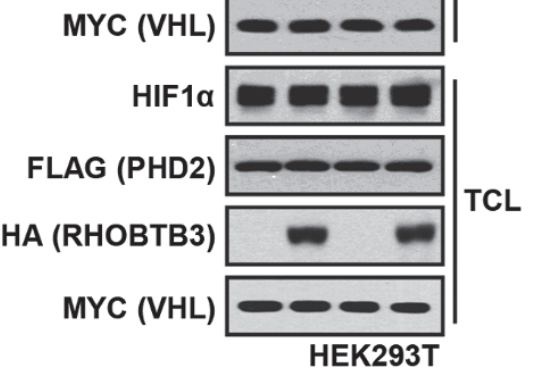


revealed that the C-terminal region of RHOBTB3 (aa 205-611) is responsible for interacting with VHL and PHD2 (Supplementary information, Figure S4A-S4C). Consistently, expression of the $\mathrm{N}$-terminal region of RHOBTB3 (aa 1-204) did not significantly affect the protein levels, transcriptional activities or levels of ubiquitination of HIF $1 \alpha$ (Figure 3D and Supplementary information, Figure S4D and S4E). Previous studies reported that two residues of RHOBTB3, asparagine 138 (N138) and aspartic acid 532 (D532), are critical for its function in regulating protein transport to the Golgi network. The N138 residue is also required for the ATPase activity of RHOBTB3 [68]. We thus tested whether alteration of the two critical residues would affect RHOBTB3's ability to downregulate HIF $\alpha$ expression. We found that the D532E mutant, but not N138D mutant, was still able to promote HIF1 $\alpha$ degradation (Supplementary information, Figure S5A and S5B), hydroxylation (Supplementary information, Figure S5C) and ubiquitination (Supplementary information, Figure S5D) and could suppress HIF1 $\alpha$ 's transcriptional activity (Supplementary information, Figure S5E), suggesting that the ATPase activity of RHOBTB3 is required. Consistently, N138D interacted with VHL and PHD2 with a much lower affinity compared with WT and D532E mutant (Supplementary information, Figure S5F and S5G).

We next asked whether RHOBTB3 could enhance the interaction between PHD2 and VHL by serving as a scaffold in the ternary complex. As shown in Supplementary information, Figure S6A, ectopically expressed PHD2 interacted weakly with VHL in the absence of RHOBTB3 in the HEK293T cells, and this interaction was enhanced upon ectopical expression of RHOBTB3. The interaction between PHD2 and VHL was significantly weaker in RHOBTB3 $3^{-/}$MEFs compared with WT MEFs (Figure $3 \mathrm{E})$. We next performed an in vitro reconstitution experiment. In vitro translated RHOBTB3 was added to the mixture containing bacterially expressed GST-PHD2 and anti-MYC-conjugated resin-bound MYC-tagged VHL. The mixture was then centrifuged to pull-down resin-bound proteins. Addition of RHOBTB3 promoted the interaction between PHD2 and VHL (Figure 3F). These results together indicate that RHOBTB3 provides a scaffold for PHD2 and VHL interaction.

We last determined whether RHOBTB 3 could serve as a scaffold for a larger complex that, in addition to PHD2 and VHL, also includes HIF $\alpha$. Indeed, we found that HIF $1 \alpha$, VHL and PHD2 were co-precipitated with RHOBTB3 (Supplementary information, Figure S6B). Importantly, overexpression of RHOBTB3 could strengthen the interaction between VHL and PHD2/ HIF1 $\alpha$ (Figure 3G). These results support the idea that RHOBTB3 amalgamates the HIF $\alpha$-VHL-RHOBTB3PHD2 complex for HIF $\alpha$ degradation.

\section{RHOBTB3 and LIMD1 form homodimers or heterodi- mers and cooperatively regulate HIF $1 \alpha$ levels \\ LIMD1 has been reported to serve as an adaptor pro- tein for PHD2 and VHL in the degradation of HIF1 $\alpha$, although if it can enhance HIF $\alpha$ hydroxylation has not been demonstrated [58]. We thus explored whether there is a functional linkage between RHOBTB3 and LIMD1. Consistent with previous reports, we found that knockdown of LIMD1, such as RHOBTB3, elevated the}

Figure 3 RHOBTB3, PHD2 and VHL form a complex. (A) RHOBTB3 interacts with endogenous PHD2 and VHL. Protein extracts of WT MEFs and RHOBTB3 ${ }^{-1-}$ MEFs (control) were immunoprecipitated with antibody against RHOBTB3 or control IgG, and analyzed by immunoblotting with antibodies indicated. (B) Ectopically expressed RHOBTB3 interacts simultaneously with PHD2 and VHL. HEK293T cells were transfected with different combinations of HA-RHOBTB3, MYC-VHL and FLAG$\mathrm{PHD} 2$. At $16 \mathrm{~h}$ post-infection, cells were lysed and the protein extracts were immunoprecipitated with antibody against HA, and the IP product was analyzed by western blotting. (C) RHOBTB3, VHL and PHD2 form a complex. HEK293T cells were transfected with HA-RHOBTB3, FLAG-PHD2 and MYC-VHL. After $16 \mathrm{~h}$, cells were harvested. Two-step co-IP was performed by first using anti-FLAG antibody, followed by elution with the FLAG peptide. The eluates were subjected to a second round of IP with anti-HA or control IgG, and the final precipitated proteins were analyzed by immunoblotting. (D) The N-terminal region of RHOBTB3 (aa 1-204) does not have a role in the degradation of HIF1 $\alpha$. RHOBTB3 $^{-1-}$ MEFs were infected with lentiviruses expressing HA-RHOBTB3 or HA-RHOBTB3 (aa 1-201). At $36 \mathrm{~h}$ post-infection, cells were maintained in normoxia or exposed to hypoxia for $8 \mathrm{~h}$, and analyzed after lysis by immunoblotting. (E) RHOBTB3 strengthens the interaction between PHD2 and VHL. RHOBTB3 ${ }^{--}$MEFs and WT MEFs were lysed and the endogenous VHL was immunoprecipitated. The IP product was analyzed by immunoblotting. (F) RHOBTB3 promotes the PHD2-VHL interaction in vitro. In vitro translated RHOBTB3 and bacterially expressed GST-PHD2 were incubated with anti-MYC-conjugated resin-bound MYC-tagged VHL. The mixtures were then pulled down by centrifugation, and analyzed by immunoblotting. (G) RHOBTB3 promotes the interaction between PHD2 and HIF1 $\alpha$. HEK293T cells were transfected with different combinations of HIF1 $\alpha$, FLAG-PHD2, MYC-VHL and HARHOBTB3. At $8 \mathrm{~h}$ post-transfection, cells were treated with $10 \mu \mathrm{M} \mathrm{MG}-132$ and maintained in normoxia or exposed to hypoxia for $10 \mathrm{~h}$. The protein extracts were immunoprecipitated with antibody against MYC (for VHL), and precipitated proteins were analyzed by immunoblotting. 
A

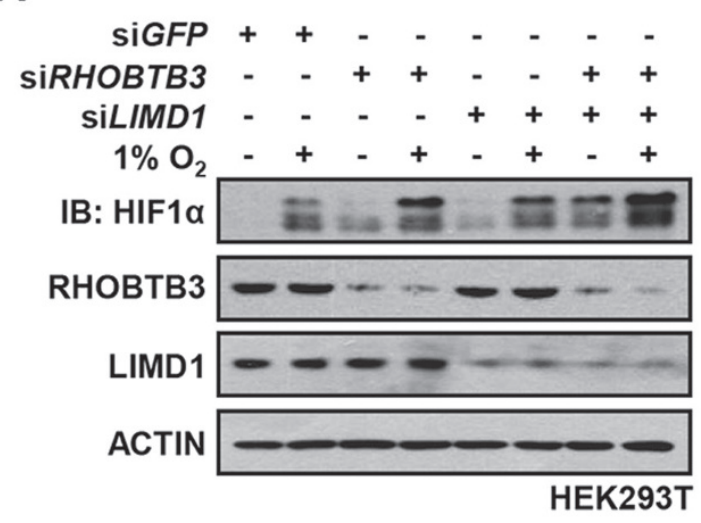

B
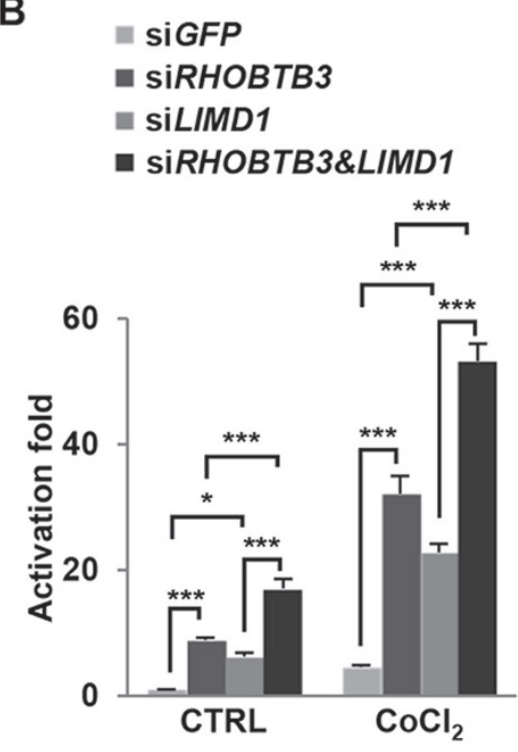

C

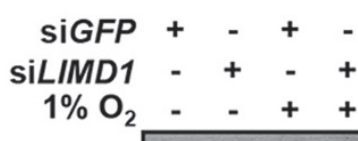

IB: HIF1 $\alpha=$

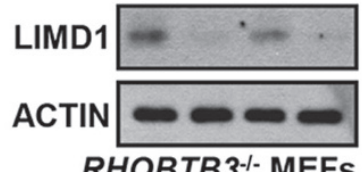

E

F
D
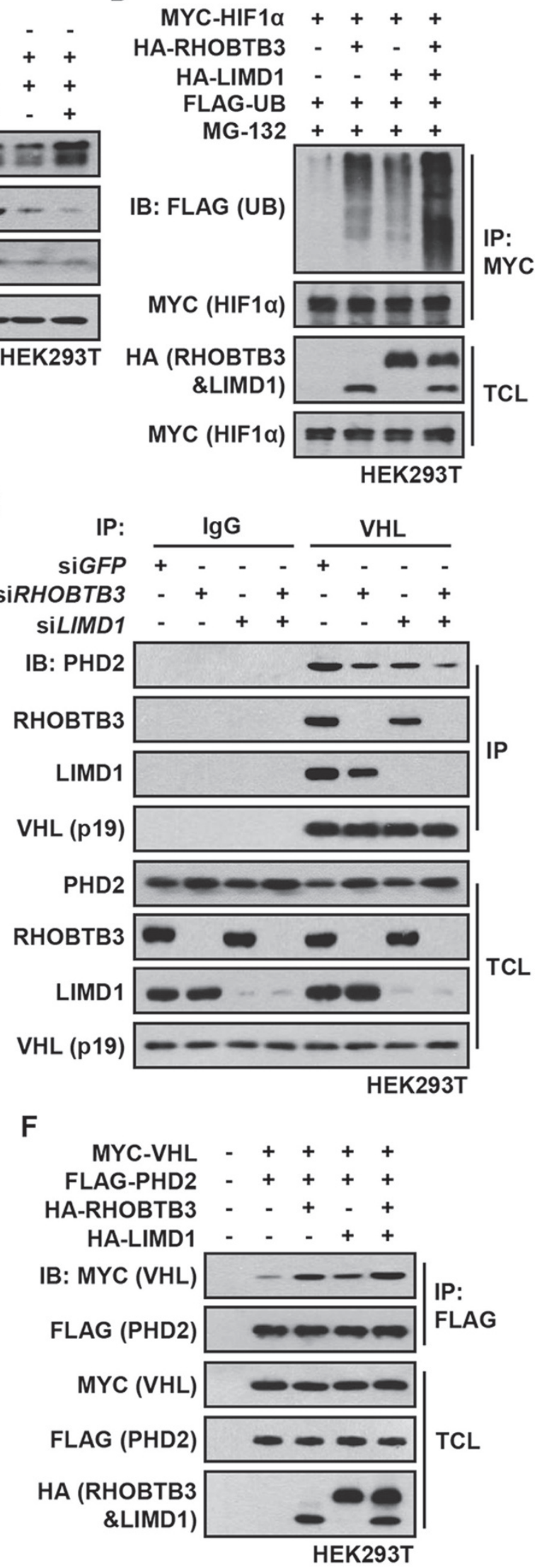
protein levels and transcriptional activities of HIF $1 \alpha$, and simultaneous knockdown of RHOBTB3 and LIMD1 led to a further increase in protein levels and transcriptional activity of HIF1 $\alpha$ (Figure 4A and 4B). Likewise, knockdown of LIMD1 in RHOBTB3 ${ }^{-/}$MEFs resulted in an increase of HIF $1 \alpha$ above the level observed in control RHOBTB3 $3^{-/}$MEFs (Figure 4C). Consistently, co-expressing RHOBTB3 and LIMD1 produced an additive effect on HIF $1 \alpha$ ubiquitination (Figure 4D). Moreover, knockdown of RHOBTB3 or LIMD1 decreased the interaction between endogenous PHD2 and VHL and knockdown of both RHOBTB3 and LIMD1 further dampened this interaction (Figure 4E). Conversely, overexpression experiment showed that RHOBTB3 and LIMD1 cooperatively strengthened this interaction (Figure 4F). These observations together suggest that both RHOBTB3 and LIMD1 are required for the suppression of HIF $1 \alpha$.

We were intrigued that RHOBTB3 and LIMD1, two proteins unrelated in sequence, could both enhance the interaction between PHD2 and VHL, and reduce HIF $\alpha$ in cells. We thus characterized the biochemistry of RHOBTB3 and LIMD1 in the context of HIF1 $\alpha$ degradation. We found that ectopically expressed MYC-tagged RHOBTB3 interacted with HA-tagged RHOBTB3 (Figure 5A), suggesting that RHOBTB3 can form homodimers. Similarly, HA-tagged LIMD1 was co-precipitated with FLAG-tagged LIMD1 (Figure 5B), indicating that LIMD1 can homodimerize. More intriguingly, when co-expressed, RHOBTB3 could be co-precipitated with LIMD1 and vice versa, indicating that RHOBTB3 can form heterodimers with LIMD1 (Figure 5A, 5B and Supplementary information, Figure S6C). In addition, endogenous LIMD1 was readily co-immunoprecipitated with RHOBTB3 (Figure 5C). Moreover, the expression of MYC-RHOBTB3 disrupted the interaction between HA-LIMD1 and FLAG-LIMD1, leading to the formation of more LIMD1-RHOBTB3 heterodimers, and addition of FLAG-LIMD1 also reduced RHOBTB3 homodimerization (Figure 5A and 5B). These results suggest that RHOBTB3 and LIMD1 prefer to heterodimerize with each other, or form a higher order complex, than to homodimerize by themselves. Moreover, knockdown of RHOBTB3 or LIMD1 reduced the interaction between endogenous LIMD1/RHOBTB3 and PHD2/VHL (Figure 4E and 5D), whereas expression of RHOBTB3 strongly promoted the interaction between PHD2/VHL and LIMD1 (Figure 5E and 5F), suggesting that the RHOBTB3-LIMD1 heterodimers are more effective scaffolds for the formation of the HIF $\alpha$ degradation complexes that contain PHD2 and VHL. Furthermore, we observed that the interaction between PHD2 and VHL (Figure 3G), and the interaction between RHOBTB3LIMD1 heterodimer and VHL/PHD2 were attenuated under hypoxic conditions (Figure 5G). The level of co-localization of RHOBTB3 and VHL was reduced under hypoxic condition (Supplementary information, Figure S3B). One obvious explanation is that RHOBTB3 and LIMD1 exert a tight control on the levels of HIF $1 \alpha$ under normoxic condition, which is eased up in hypoxia to allow for appropriate HIF $\alpha$ accumulation. Notably, although LIMD1 and RHOBTB3 cooperatively regulate HIF1 $\alpha$ levels, LIMD1, unlike RHOBTB3, does not promote the hydroxylation on the proline-564 residue of HIF $1 \alpha$ as RHOBTB3 does (Supplementary information, Figure S6D and S6E).

Figure 4 RHOBTB3 and LIMD1 cooperatively regulate HIF1 $\alpha$. (A) RHOBTB3 and LIMD1 cooperatively suppress the protein level of HIF1 $\alpha$. HEK293T cells were infected with lentiviruses expressing siRNA targeting GFP, RHOBTB3 and/or LIMD1. At $16 \mathrm{~h}$ post-infection, cells were maintained in normoxia or exposed to hypoxia for $8 \mathrm{~h}$ before lysis and immunoblotting with antibodies indicated. (B) RHOBTB3 and LIMD1 cooperatively suppress the transcriptional activities of HIF1 $\alpha$. HEK293T cells were infected with different combinations of lentiviruses as indicated. Transcriptional activities of HIF $1 \alpha$ were measured using a dual luciferase assay system as described in Figure 2E. Data are presented as mean $\pm \mathrm{SEM}, n=3$ for each group, ${ }^{*} P<0.05$, ${ }^{* *} P<0.001$ (ANOVA followed by Tukey). (C) Knockdown of LIMD1 in RHOBTB3 ${ }^{-1-}$ MEFs further increases the protein levels of HIF1 $\alpha$. RHOBTB3 ${ }^{-/-}$MEFs were infected with lentiviruses expressing siRNA targeting GFP or LIMD1. At $36 \mathrm{~h}$ post-infection, cells were maintained in normoxia or exposed to hypoxia for $8 \mathrm{~h}$, before the western blot analysis. (D) RHOBTB3 and LIMD1 cooperatively promote the ubiquitination of HIF1 $\alpha$. HEK293T cells were transfected with different combinations of MYC-HIF1 $\alpha$, HA-RHOBTB3, HA-LIMD1 and FLAG-UB (ubiquitin). After treatment with $10 \mu \mathrm{M}$ MG-132 for $10 \mathrm{~h}$, the cells were lysed, and the lysates were subjected to IP with antibody against MYC (for HIF1 $\alpha$ ). The IP product was analyzed by western blotting to determine the ubiquitination levels of HIF1 $\alpha$. (E) Knockdown of RHOBTB3 and/or LIMD1 decreases PHD2-VHL interaction. HEK293T cells were infected with lentiviruses expressing siRNA targeting GFP, RHOBTB3 and/or LIMD1. At 16 h post-infection, cells were lysed and the endogenous VHL was immunoprecipitated, and the IP product was analyzed by immunoblotting. (F) Ectopically expressed RHOBTB3 and LIMD1 cooperatively promote PHD2-VHL interaction. HEK293T cells were transfected with different combinations of MYC-VHL, HA-RHOBTB3, HA-LIMD1 and FLAG-PHD2. Protein extracts from the transfected cells were subjected to IP with antibody against FLAG and analyzed by immunoblotting with antibodies indicated. 
A

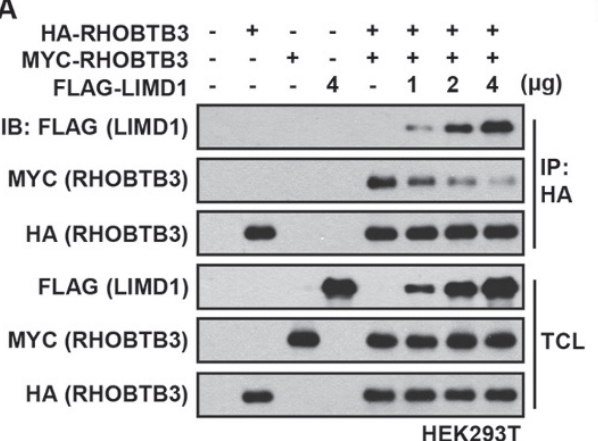

D

C

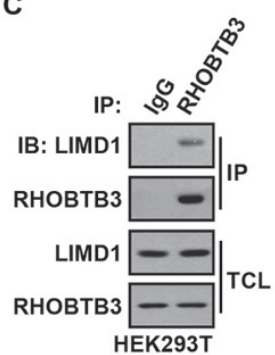

$\mathbf{F}$

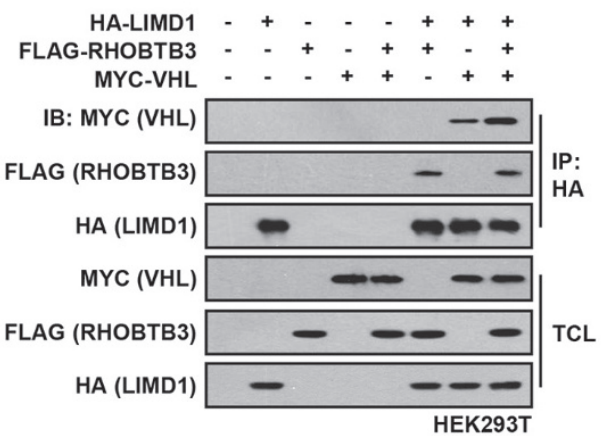

B siGFP + - + siLIMD1 - + - +

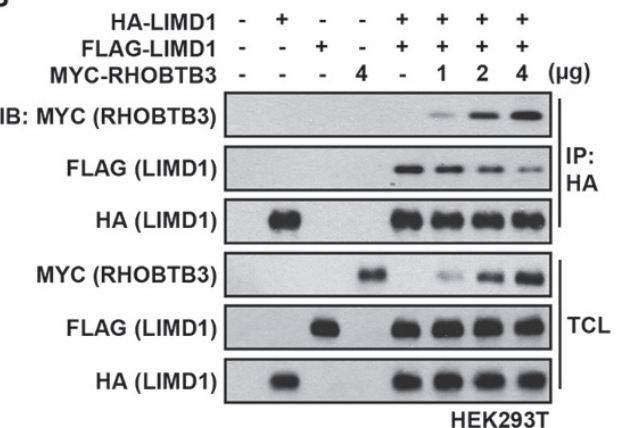

$E$ IB: PHD2 $\longrightarrow$ VHL (p19)
RHOBTB3 RHOBTB3 $\square$
PHD2 $-\infty-\infty$
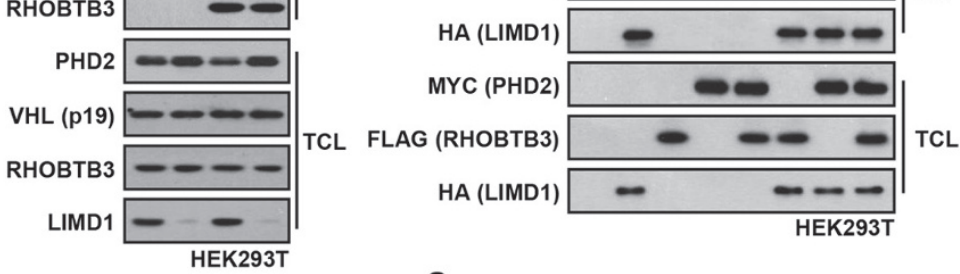

G

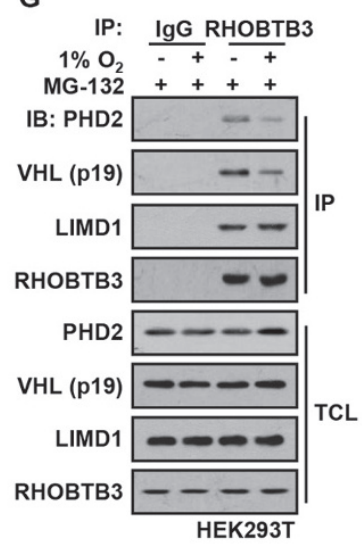

Figure 5 Dimerization of RHOBTB3 and LIMD1. (A) Ectopically expressed HA-tagged RHOBTB3 interacts with MYC-tagged RHOBTB3 or FLAG-tagged LIMD1. HEK293T cells were transfected with different combinations of HA-RHOBTB3, MYCRHOBTB3 and FLAG-LIMD1. Cells were then lysed and the protein extracts were immunoprecipitated with antibody against HA. The IP product was analyzed by immunoblotting. (B) Ectopically expressed HA-tagged LIMD1 interacts with FLAGtagged LIMD1 or MYC-tagged RHOBTB3. Lysates from transfected cells were subjected to IP with antibody against HA (for LIMD1), and analyzed by immunoblotting as in A. (C) RHOBTB3 interacts with endogenous LIMD1. Lysates of HEK293T cells were immunoprecipitated with antibody against RHOBTB3 or IgG (control), and analyzed by immunoblotting using antibodies indicated. (D) Knockdown of LIMD1 attenuates the interaction between RHOBTB3 and PHD2/VHL. HEK293T cells were infected with lentivirus expressing siRNA targeting GFP or LIMD1. At $16 \mathrm{~h}$ post-infection, cells were lysed and the endogenous RHOBTB3 was then immunoprecipitated, and analyzed by immunoblotting with antibodies indicated. (E, F) Ectopically expressed RHOBTB3 promotes the interaction between LIMD1 and PHD2 (E), and the interaction between LIMD1 and VHL (F). HEK293T cells were transfected with different combinations of HA-LIMD1, FLAG-RHOBTB3, MYC-PHD2 (E) and MYC-VHL (F). Protein extracts were immunoprecipitated and analyzed by immunoblotting. (G) Hypoxia attenuates the interaction between endogenous RHOBTB3/LIMD1 and PHD2/VHL. HEK293T cells were maintained in normoxia or exposed to hypoxia for $8 \mathrm{~h}$ in presence of $10 \mu \mathrm{M}$ MG-132 to prevent the degradation of VHL under hypoxic condition as described previously [80]. Endogenous RHOBTB3 was then immunoprecipitated, and the IP product was analyzed by immunoblotting with antibodies indicated. 
RHOBTB3 suppresses the Warburg effect and inhibits tumorigenesis

We next examined whether RHOBTB3 could suppress the Warburg effect since it could significantly reduce HIF $\alpha$ levels in cells, and levels of HK2, LDHA, GLUT1 and PDK1, known stream targets of HIFs, were elevated in $\mathrm{RHOBTB}^{-/-}$MEFs under both normoxic and hypoxic conditions (Figure 6A and 6B). The Warburg effect is characterized by high rates of glucose uptake and lactate production regardless of oxygen concentration [30]. We thus measured the glucose consumption and lactate production rates, and found that they were increased in RHOBTB $3^{-/-}$MEFs in both normoxia and hypoxia (Figure $6 \mathrm{C}$ and $6 \mathrm{D}$ ). We also explored the possibility that RHOBTB3 and its partner LIMD1 could regulate the Warburg effect cooperatively, and found that knockdown of RHOBTB3 or LIMD1 promoted glucose consumption and lactate production under both normoxic and hypoxic conditions, and their double knockdown in HEK293T cells further enhanced these effects (Figure 6E and 6F). Taken together, our data suggest that RHOBTB3 inhibits the Warburg effect most likely through promoting HIF degradation.

Finally, we explored whether regulation of HIF $\alpha$ by RHOBTB3 has any relevance to tumorigenesis. A search in three independent public data sets of kidney cancer: Yusenko Renal (GEO data set GSE11151) [74], Beroukhim Renal (GEO data set GSE14994) [75] and Gumz Renal (GEO data set GSE6344) [76], summarized by The Oncomine Platform (Life Technologies, Ann Arbor, MI, USA) reveal that the mRNA levels of RHOBTB3 are significantly decreased in clear cell renal cell carcinoma, papillary renal cell carcinoma, hereditary clear cell renal cell carcinoma and non-hereditary clear cell renal cell carcinoma subtypes (Figure 7A). None of the data sets deposited in Oncomine show a significant upregulation of RHOBTB3 in kidney cancer (Figure 7A). These results suggest a potential role of RHOBTB3 in suppressing tumorigenesis. To test this hypothesis, we performed xenograft experiment implanting subcutaneously Ras V12/E1A H133-transformed RHOBTB $3^{-/-}$MEFs and, as a control, WT MEFs, into nude mice. Xenografts derived from $R H O B T B 3^{-/}$MEFs were significantly larger in both volume and weight when compared with those derived from control MEFs (Figure 7B and Supplementary information, Figure S7A). In addition, these xenografts had elevated levels of HIF $1 \alpha$ and its targets including GLUT1, HK2, LDHA, PHD2 and carbonic anhydrase IX (CA9) (Figure 7C and Supplementary information, Figure S7B). Moreover, sections from RHOBTB3 null xenografts showed enhanced staining for downstream targets of HIF (Supplementary information, Figure S7C).
Furthermore, the xenografts derived from RHOBTB3-deficient (siRHOBTB3) HeLa cells showed increased growth rates, tumor volumes, tumor weights and deregulated HIF signaling (Figure 7D, 7E and Supplementary information, Figure S7D-S7F), whereas HeLa cells overexpressing RHOBTB3 were unable to form xenograft tumors (Supplementary information, Figure S7G). We also detected higher proliferation rates in $\mathrm{RHOBTB}^{-/-} \mathrm{MEFs}$ compared with WT MEFs, which could be reduced by the knockdown of HIF $1 \alpha$, suggesting that RHOBTB3 suppresses cell proliferation, at least in part, through HIFs (Supplementary information, Figure S7H). Thus, it is reasonable to conclude that RHOBTB3 suppresses tumorigenesis through downregulating HIF $\alpha$ levels.

\section{Discussion}

In this study, we show that RHOBTB3 is essential for the downregulation of HIF $\alpha$. RHOBTB3 promotes the hydroxylation, ubiquitination and degradation of HIF $\alpha$. Mechanistically, RHOBTB3 simultaneously interacts with PHD and VHL, serving as a scaffold for the assembly of the RHOBTB3-PHD-VHL-HIF $\alpha$ degradation complex. Within the complex, PHD2 exhibits increased hydroxylase activity towards HIF $\alpha$, resulting in enhanced interaction between HIF $\alpha$ and VHL, owing to the higher affinity of VHL for hydroxylated HIF $\alpha$. This facilitates the channeling of hydroxylated HIF $\alpha$ to the VHL-E3 ligase complex for ubiquitination and subsequent degradation. Remarkably, RHOBTB3 is able to form heterodimer with LIMD1, resulting in maximal degradation of HIF (Figure 7F). Although PHD2 appears to have a major role in the RHOBTB3-enhanced hydroxylation of HIF1 $\alpha$, the other PHD isoforms may also participate in the process.

While RHOBTB3 is uniquely involved in facilitating hydroxylation of HIF, RHOBTB3 and LIMD1 are both critical for the degradation of HIF $\alpha$ by scaffolding PHD2 and VHL. Evidence for their mutual compensation is the observation that double knockdown of RHOBTB3 and LIMD1 increases the accumulation of HIF $1 \alpha$ above the level in single knockdown under both normoxic and hypoxic conditions. In addition, knockdown of LIMD1 in RHOBTB $3^{-/-}$MEFs further elevates the protein levels of HIF $1 \alpha$. Moreover, while overexpression of RHOBTB3 or LIMD1 alone can strengthen PHD2-VHL interaction and promote HIF $1 \alpha$ degradation, overexpression of both proteins can further strengthen PHD2-VHL interaction and cause additional reduction in the level of HIF $\alpha$. These observations also support the notion that RHOBTB3/ LIMD1 heterodimers are more efficient than homodimers of each protein in strengthening PHD2-VHL interaction 
A

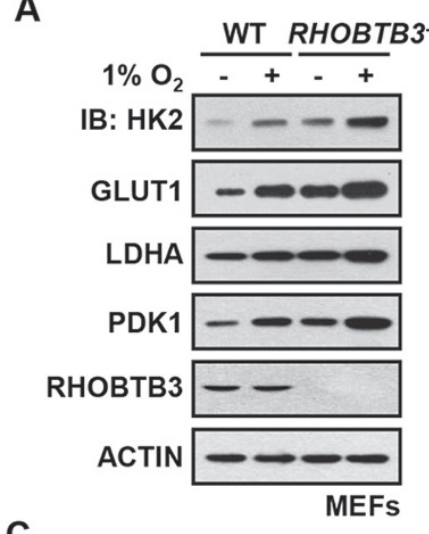

C

WT

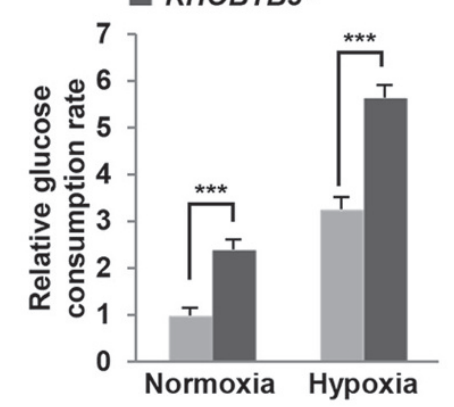

E

$$
\begin{aligned}
& \text { " siGFP } \\
& \text { " siRHOBTB3 } \\
& \text { " siLIMD1 } \\
& \text { - siRHOBTB3\&LIMD1 }
\end{aligned}
$$

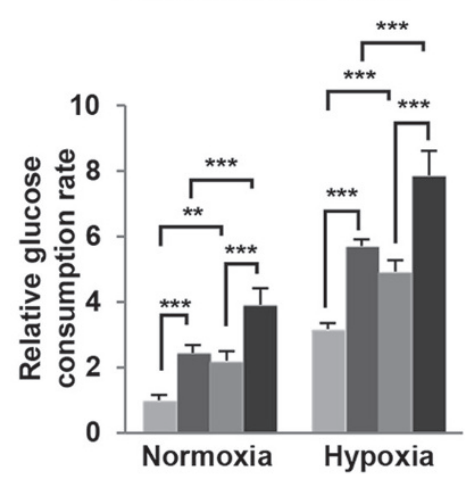

B

$$
\begin{aligned}
& \text { " WT Nomoxia }
\end{aligned}
$$

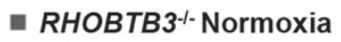

$$
\begin{aligned}
& \text { - WT Hypoxia } \\
& \text { - RHOBTB }{ }^{-1-} \text { Hypoxia }
\end{aligned}
$$

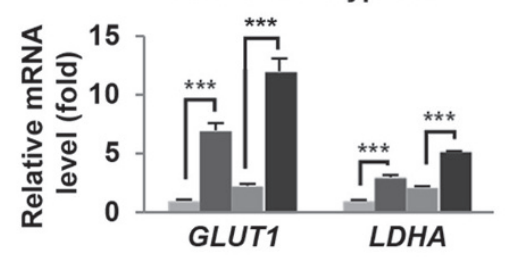

D

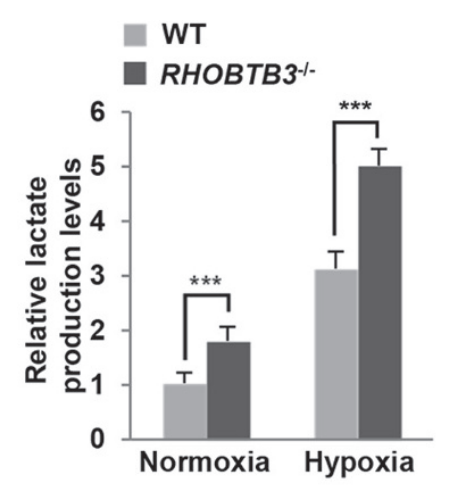

$\mathbf{F}$

$$
\begin{aligned}
& \text { siGFP } \\
& \text { siRHOBTB3 } \\
& \text { siLIMD1 }
\end{aligned}
$$

- siRHOBTB3\&LIMD1

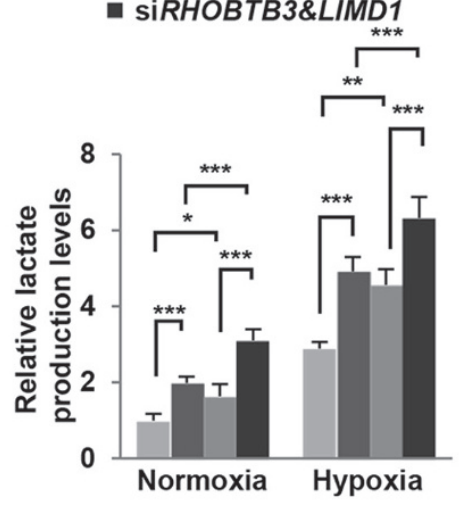

Figure 6 RHOBTB3 is a negative regulator of the Warburg effect. (A) Knockout of RHOBTB3 elevates the expression of HK2, LDHA GLUT1 and PDK1. RHOBTB3 ${ }^{-1-}$ MEFs and WT MEFs were maintained in normoxia or exposed to hypoxia for $16 \mathrm{~h}$. Cells were then lysed and the protein extracts were analyzed by immunoblotting with antibodies indicated. (B) RHOBTB3 deficiency leads to in-

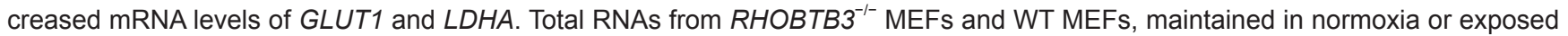
to hypoxia for $16 \mathrm{~h}$, were purified, and analyzed by real-time PCR analysis for the expression levels of GLUT1 and LDHA. Values are presented as mean \pm SEM, $n=3$ for each group, three replicate experiments. ${ }^{* * *} P<0.001$ (ANOVA followed by Tukey). (C, D)

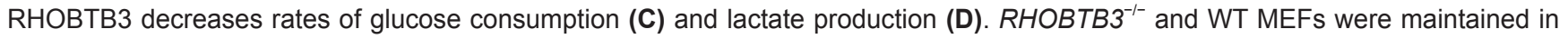
normoxia or exposed to hypoxia for $8 \mathrm{~h}$, and glucose consumption rates (C) and lactate production rates (D) were measured. Values are presented as mean \pm SEM, $n=3$ for each group, ${ }^{* * *} P<0.001$ (ANOVA followed by Tukey). (E, F) RHOBTB3 and LIMD1 cooperatively decrease glucose consumption (E) and lactate production (F). HEK293T cells were infected with lentiviruses expressing siRNAs targeting GFP, RHOBTB3 and/or LIMD1. At $16 \mathrm{~h}$ post-infection, cells were maintained in normoxia or exposed to hypoxia for $8 \mathrm{~h}$ and glucose consumption rates $(\mathrm{E})$ and lactate production rates $(\mathrm{F})$ were measured. Values are presented as mean $\pm \mathrm{SEM}, n=3$ for each group, ${ }^{*} P<0.05,{ }^{* *} P<0.01,{ }^{* * *} P<0.001$ (ANOVA followed by Tukey). 
A

Gumz
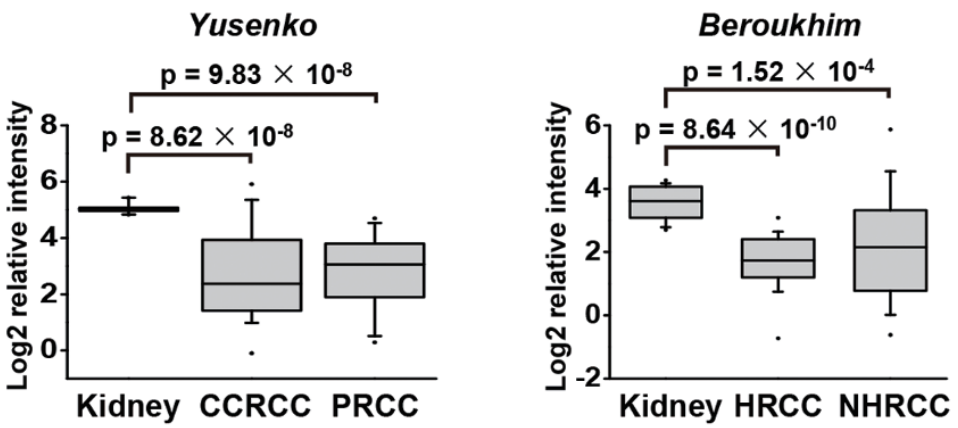

C
B

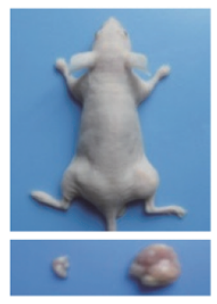

WT RHOBTB3 ${ }^{-/}$

$n=5, P<0.0001$

D
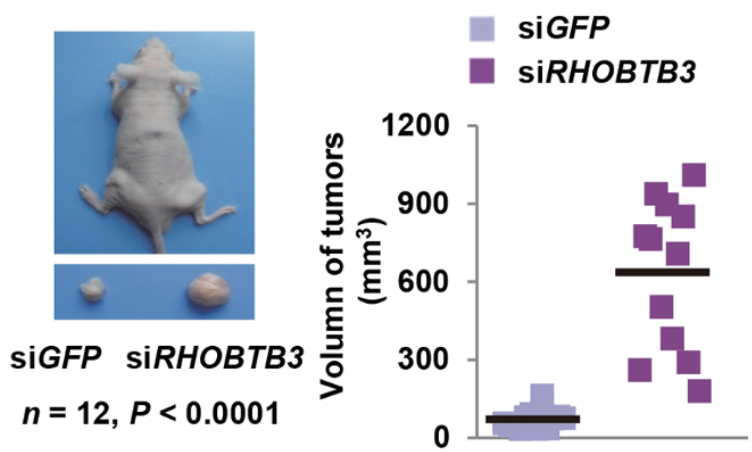

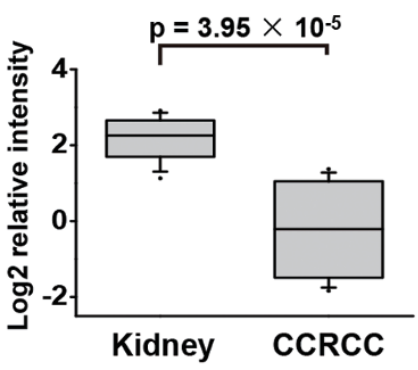

C

IB: HIF1a

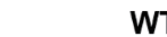

RHOBTB3 $^{-1-}$

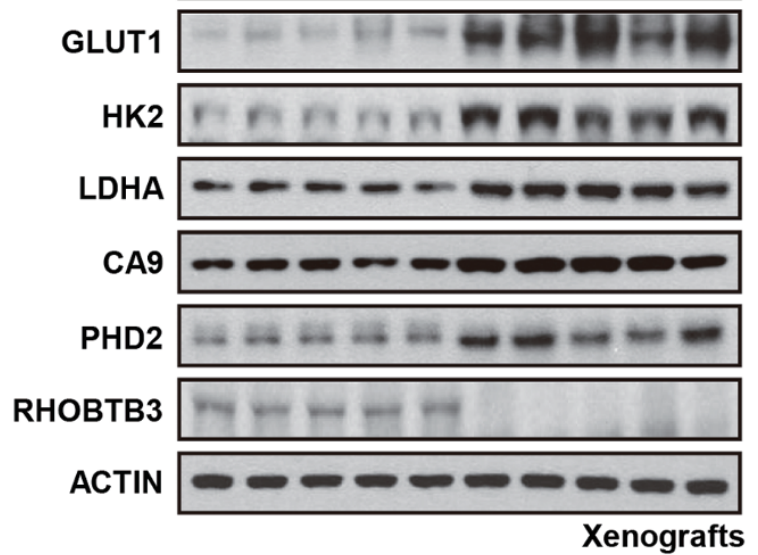

$\mathbf{E}$

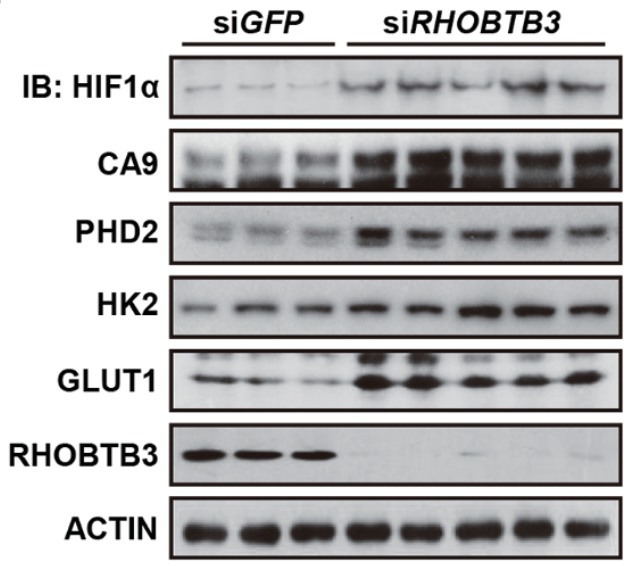

Xenografts

$\mathbf{F}$

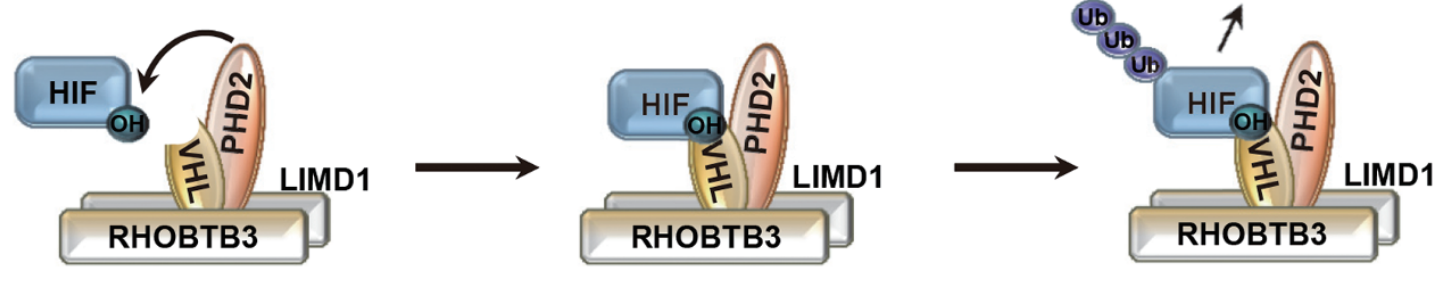

Cell Research | Vol 25 No 9 | September 2015 
and degrading HIF1 $\alpha$. In physiological settings where both RHOBTB3 and LIMD1 are present, a RHOBTB3/ LIMD1-PHD2-VHL-HIF $\alpha$ degradation complex is assembled to maximize the control of HIF $\alpha$ levels. It is also noteworthy that the interaction between RHOBTB3/ LIMD1 and PHD2/VHL is loosened under hypoxic conditions, allowing the appropriate accumulation of HIF $\alpha$ that is required for the adaptive responses to low-oxygen stresses.

It has been established that RHOBTB3 is a component of a CUL3-dependent E3 ubiquitin ligase complex and required for degradation of cyclin E and MUF-1 [65-67]. The present study implicates RHOBTB3 in the degradation of HIF $\alpha$, which is mediated by VHL, a member of CUL2-dependent E3 ubiquitin ligase complex [53, 54]. It is interesting to note that RHOBTB3 can function in both CUL2- and CUL3-dependent ubiquitination pathways. These two pathways might be parallel, or intertwine; further investigation is needed to test whether CUL3 also cooperates with RHOBTB3/VHL in HIF $\alpha$ regulation. Furthermore, we show that HSP90, acting as a chaperon of HIF $1 \alpha$ and PHD2 as reported previously [71-73], can indirectly associate with the RHOBTB3-PHD2-VHL complex through HIF1 $\alpha$.

Also interesting is the reduction in the expression levels of RHOBTB3 in nearly all RCC data sets collected on Oncomine Platform. Our experimental data identify RHOBTB3 as a potent suppressor of the Warburg effect, repressing the production of lactic acid and glucose consumption rates. RHOBTB3 also markedly inhibits xenograft growth in nude mice. Taken together, our work demonstrates that RHOBTB3 assembles a degradation complex for HIF $\alpha$, suppresses Warburg effect and func- tions as a critical tumor suppressor.

\section{Materials and Methods}

\section{Generation of RHOBTB3 ${ }^{-/-}$MEFs}

$R H O B T B 3^{-/}$mice were obtained from Knockout Mouse Project (KOMP) Repository (RHOBTB $3^{\text {tmla(KOMP)Wtsi }}$, project ID:

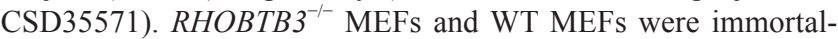
ized by introducing SV40 T antigen (for cellular experiments) or Ras (V12) and E1A (H133) oncoproteins (for xenograft model) into primary MEFs from $R H O B T B 3^{-/-}$mice and their WT littermates.

Cell culture, transient transfection, and lentivirus packaging

HEK293T and MEFs were maintained in Dulbecco's modified Eagle's medium (DMEM) supplemented with $10 \%$ fetal bovine serum (FBS), $2 \mathrm{mM}$ L-glutamine, $100 \mathrm{IU}$ penicillin, $100 \mathrm{mg} / \mathrm{ml}$ streptomycin at $37{ }^{\circ} \mathrm{C}$ in a humidified incubator containing $5 \%$ $\mathrm{CO}_{2}$. Polyethylenimine (Cat. \#23966, Polysciences, Inc.) at a final concentration of $10 \mu \mathrm{M}$ was used to transfect HEK293T cells. Total DNA for each plate was adjusted to the same amount by adding relevant empty vector. Lentiviruses for infection of the MEFs were packaged in HEK293T cells after transfection using Lipofectamine 2000 (Cat. 11668-027, Invitrogen). At $30 \mathrm{~h}$ post-transfection, medium was collected for further infection.

\section{Immunoprecipitation and immunoblotting}

Cell lysis and IP were carried out as previously described [77]. Briefly, cells were lysed in a lysis buffer containing $20 \mathrm{mM}$ Tris$\mathrm{HCl}(\mathrm{pH} 7.5), 150 \mathrm{mM} \mathrm{NaCl}, 1 \mathrm{mM}$ EDTA, 1 mM EGTA, $1 \%$ Triton X-100, $2.5 \mathrm{mM}$ sodium pyrophosphate, $1 \mathrm{mM} \beta$-glycerolphosphate, $1 \mathrm{mM}$ sodium orthovanadate and a protease inhibitor cocktail. Cell lysates were incubated with respective antibodies overnight. Protein aggregates resulting from the overnight incubation were removed by centrifugation, and protein $\mathrm{A} / \mathrm{G}$ beads were then added into the lysates and incubated for another $3 \mathrm{~h}$. Protein

Figure 7 RHOBTB3 suppresses tumorigenesis. (A) Expression of RHOBTB3 in human renal cancer samples in public data

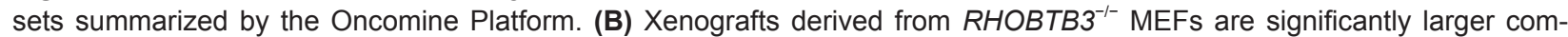
pared with those derived from control MEFs. Ras V12/E1A H133-transformed RHOBTB3 ${ }^{-1-}$ MEFs and control WT MEFs $(1 \times$ $10^{6}$ ) were injected intradermally into each flank of nude mice. Tumors were allowed to develop for 35 days. Tumor volumes were then determined by direct measurement using a caliper and calculated by the formula: (widest diameter $\times$ smallest diameter $\left.^{2}\right) / 2$. Data were presented as mean \pm SEM, $n=5$ for each group, $P<0.0001$ (Student's $t$-test). (C) The protein levels of HIF $1 \alpha$ and its targets are upregulated in xenografts derived from $\mathrm{RHOBTB}^{-/-} \mathrm{MEFs}$. Xenografts derived from $\mathrm{RHOBTB}^{-/-}$ and WT MEFs as described in B were homogenized and analyzed by immunoblotting with the indicated antibodies. (D) Xenografts derived from $R H O B T B 3$ knocked down HeLa cells are significantly larger compared with those derived from control cells. Suspensions of HeLa cells expressing siGFP or siRHOBTB3 $\left(1 \times 10^{6}\right.$ each) were injected intradermally into each flank of nude mice. Tumors were allowed to develop for 37 days and their volumes were calculated as described in Figure 7B. The values of tumor volumes are presented as mean \pm SEM, $n=12$ for each group, $P<0.0001$ (Student's $t$-test). (E) Protein levels of HIF1 $\alpha$ and its target genes are upregulated in xenografts of RHOBTB3 knocked down HeLa cells. Xenografts derived from HeLa-siGFP or HeLa-siRHOBTB3 cells were homogenized and analyzed by immunoblotting with antibodies indicated. (F) Simplified model depicting that RHOBTB3 and LIMD1 promote the formation of the HIF1 $\alpha$ degradation complex. In this scheme, RHOBTB3 and LIMD1 form a heterodimer, which interacts with PHD2 and VHL, and recruits HIF $\alpha$, forming a RHOBTB3/LIMD1-PHD2-VHL-HIF $\alpha$ complex that promotes the hydroxylation, ubiquitination and degradation of HIF $\alpha$. Hypoxia loosens the interaction between RHOBTB3-LIMD1 and HIF $\alpha$-VHL-PHD2, allowing for the accumulation of HIF $\alpha$ in cells under hypoxia. Notably, RHOBTB3 can directly promote PHD2-mediated hydroxylation of HIF $\alpha$, whereas LIMD1 cannot. 
levels were analyzed on gels. For ubiquitination assays, cells were lysed in RIPA buffer containing 1\% SDS and boiled. The protein extracts were diluted using RIPA buffer without SDS to a final concentration of $0.2 \%$ SDS, and were subjected to IP. The IP product was analyzed by immunoblotting (IB).

\section{Luciferase reporter assays}

Cells cultured in 12-well plates were transfected with the indicated plasmids, washed with phosphate-buffered saline, and lysed in Reporter Lysis Buffer (Invitrogen). Luciferase reporter activities were measured in triplicate using the Dual-Luciferase Reporter Assay System (Promega) according to the manufacturer's protocol and quantified using a GloMax 96-well plate luminometer (Promega). The firefly luciferase to Renilla luciferase ratios were determined and defined as the relative luciferase activity.

\section{Xenograft model}

Five-week-old male athymic BALB/c nude mice were housed under specific pathogen-free conditions in compliance with the Institutional Animal Care and Use Committee at Xiamen University. Single cell suspension of HeLa cells infected with lentivirus expressing siRNAs targeting RHOBTB3 or control (siGFP), HeLa cells stably expressing RHOBTB3, or Ras V12/E1A-transformed WT and $R H O B T B 3^{--}$MEFs in $0.1 \mathrm{ml}$ DMEM without FBS were injected intradermally into each flank of nude mice. The xenografts were then measured as described in figure legends.

\section{In vitro hydroxylation assay}

In vitro hydroxylation assay was performed as described previously $[78,79]$. Briefly, cells were lysed in HEB buffer $(20 \mathrm{mM}$ Tris- $\mathrm{HCl}, \mathrm{pH} 7.4,5 \mathrm{mM} \mathrm{KCl}, 1.5 \mathrm{mM} \mathrm{MgCl}_{2}$ ) containing a protease inhibitor cocktail. Two-hundred microgram of total protein from each cell lysate was used for each reaction. The cell lysates were mixed with resin-bound HIF1 $\alpha$ (aa 401-603) or its P564A mutant in NETN buffer (20 mM Tris, $\mathrm{pH} 8.0,100 \mathrm{mM} \mathrm{NaCl}, 1$ mM EDTA, $0.5 \%$ Nonidet P-40 and $1 \mathrm{mM}$ phenylmethylsulfonyl fluoride) containing $2 \mathrm{mM}$ ascorbic acid, $100 \mu \mathrm{M} \mathrm{FeSO}_{4}$ and 5 $\mathrm{mM} \alpha$-ketoglutarate in a final volume of $200 \mu \mathrm{l}$. After mild agitation $(2500 \times \mathrm{g})$ for $90 \mathrm{~min}$ at $30{ }^{\circ} \mathrm{C}$, the reaction mixtures were centrifuged and washed three times in five volumes of NETN buffer. The final resin-bound proteins were dissolved in an equal volume of $2 \times$ SDS buffer, followed by IB with antibodies indicated.

\section{Statistical analyses}

ANOVA with Tukey's post-test was used to compare values among different experimental groups using the SPSS Statistics 17.0 program. For experiments with only two groups, Student's $t$-test was used as specified in the figure legends.

\section{Acknowledgments}

We thank colleagues and members of SCL laboratory for suggestions and technical assistance and Dr HR Wang for useful discussions. This work was supported by the National Natural Science Foundation of China (31130016, 31221065, 31370744 and 31300626), the National Basic Research Program of China (973 Program; 2014CB910602, 2011CB910800 and 2013CB530600) and the National Science Foundation for Fostering Talents in Basic Research of the National Natural Science Foundation of China
(J1310027/J0106). S-CL is a Cheung Kong Scholar.

\section{References}

1 Majmundar AJ, Wong WJ, Simon MC. Hypoxia-inducible factors and the response to hypoxic stress. Mol Cell 2010; 40:294-309.

2 Kaelin WG Jr, Ratcliffe PJ. Oxygen sensing by metazoans: the central role of the HIF hydroxylase pathway. Mol Cell 2008; 30:393-402.

3 Semenza GL. Hypoxia-inducible factors in physiology and medicine. Cell 2012; 148:399-408.

4 Semenza GL. Oxygen sensing, homeostasis, and disease. $N$ Engl J Med 2011; 365:537-547.

5 Semenza GL, Jiang BH, Leung SW, et al. Hypoxia response elements in the aldolase A, enolase 1, and lactate dehydrogenase A gene promoters contain essential binding sites for hypoxia-inducible factor 1. J Biol Chem 1996; 271:3252932537.

6 Wang GL, Semenza GL. Purification and characterization of hypoxia-inducible factor 1 . J Biol Chem 1995; 270:12301237.

7 Wang GL, Jiang BH, Rue EA, Semenza GL. Hypoxia-inducible factor 1 is a basic-helix-loop-helix-PAS heterodimer regulated by cellular $\mathrm{O}_{2}$ tension. Proc Natl Acad Sci USA 1995; 92:5510-5514.

8 Wang GL, Semenza GL. Characterization of hypoxia-inducible factor 1 and regulation of DNA binding activity by hypoxia. J Biol Chem 1993; 268:21513-21518.

9 Jiang BH, Rue E, Wang GL, Roe R, Semenza GL. Dimerization, DNA binding, and transactivation properties of hypoxia-inducible factor 1. J Biol Chem 1996; 271:17771-17778.

10 Bertout JA, Patel SA, Simon MC. The impact of $\mathrm{O}_{2}$ availability on human cancer. Nat Rev Cancer 2008; 8:967-975.

11 Erler JT, Bennewith KL, Nicolau M, et al. Lysyl oxidase is essential for hypoxia-induced metastasis. Nature 2006; 440:1222-1226.

12 Staller P, Sulitkova J, Lisztwan J, Moch H, Oakeley EJ, Krek W. Chemokine receptor CXCR4 downregulated by von Hippel-Lindau tumour suppressor pVHL. Nature 2003; 425:307311.

13 Shweiki D, Itin A, Soffer D, Keshet E. Vascular endothelial growth factor induced by hypoxia may mediate hypoxia-initiated angiogenesis. Nature 1992; 359:843-845.

14 Gordan JD, Thompson CB, Simon MC. HIF and c-Myc: sibling rivals for control of cancer cell metabolism and proliferation. Cancer Cell 2007; 12:108-113.

15 Wouters BG, Koritzinsky M. Hypoxia signalling through $\mathrm{mTOR}$ and the unfolded protein response in cancer. Nat Rev Cancer 2008; 8:851-864.

16 Keith B, Johnson RS, Simon MC. HIF1alpha and HIF2alpha: sibling rivalry in hypoxic tumour growth and progression. Nat Rev Cancer 2012; 12:9-22.

17 Forsythe JA, Jiang BH, Iyer NV, et al. Activation of vascular endothelial growth factor gene transcription by hypoxia-inducible factor 1. Mol Cell Biol 1996; 16:4604-4613.

18 Boutin AT, Weidemann A, Fu Z, et al. Epidermal sensing of oxygen is essential for systemic hypoxic response. Cell 2008; 133:223-234. 
19 Wang GL, Semenza GL. General involvement of hypoxia-inducible factor 1 in transcriptional response to hypoxia. Proc Natl Acad Sci USA 1993; 90:4304-4308.

20 Iyer NV, Kotch LE, Agani F, et al. Cellular and developmental control of $\mathrm{O}_{2}$ homeostasis by hypoxia-inducible factor 1 alpha. Genes Dev 1998; 12:149-162.

21 Ebert BL, Firth JD, Ratcliffe PJ. Hypoxia and mitochondrial inhibitors regulate expression of glucose transporter-1 via distinct Cis-acting sequences. J Biol Chem 1995; 270:2908329089.

22 Firth JD, Ebert BL, Ratcliffe PJ. Hypoxic regulation of lactate dehydrogenase A. Interaction between hypoxia-inducible factor 1 and cAMP response elements. J Biol Chem 1995; 270:21021-21027.

23 Mathupala SP, Rempel A, Pedersen PL. Glucose catabolism in cancer cells: identification and characterization of a marked activation response of the type II hexokinase gene to hypoxic conditions. J Biol Chem 2001; 276:43407-43412.

24 Papandreou I, Cairns RA, Fontana L, Lim AL, Denko NC. HIF-1 mediates adaptation to hypoxia by actively downregulating mitochondrial oxygen consumption. Cell Metab 2006; 3:187-197.

25 Kim JW, Tchernyshyov I, Semenza GL, Dang CV. HIF-1-mediated expression of pyruvate dehydrogenase kinase: a metabolic switch required for cellular adaptation to hypoxia. Cell Metab 2006; 3:177-185.

26 Sugden MC, Holness MJ. Recent advances in mechanisms regulating glucose oxidation at the level of the pyruvate dehydrogenase complex by PDKs. Am J Physiol Endocrinol Metab 2003; 284:E855-E862.

27 Seagroves TN, Ryan HE, Lu H, et al. Transcription factor HIF-1 is a necessary mediator of the pasteur effect in mammalian cells. Mol Cell Biol 2001; 21:3436-3444.

28 Semenza GL. HIF-1 mediates metabolic responses to intratumoral hypoxia and oncogenic mutations. J Clin Invest 2013; 123:3664-3671.

29 Warburg O. On respiratory impairment in cancer cells. Science $1956 ;$ 124:269-270.

30 Vander Heiden MG, Cantley LC, Thompson CB. Understanding the Warburg effect: the metabolic requirements of cell proliferation. Science 2009; 324:1029-1033.

31 Zhao F, Mancuso A, Bui TV, et al. Imatinib resistance associated with BCR-ABL upregulation is dependent on HIF-1alpha-induced metabolic reprograming. Oncogene 2010; 29:2962-2972.

32 Brugarolas J, Kaelin WG Jr. Dysregulation of HIF and VEGF is a unifying feature of the familial hamartoma syndromes. Cancer Cell 2004; 6:7-10.

33 Rankin EB, Giaccia AJ. The role of hypoxia-inducible factors in tumorigenesis. Cell Death Differ 2008; 15:678-685.

34 Klimova T, Chandel NS. Mitochondrial complex III regulates hypoxic activation of HIF. Cell Death Differ 2008; 15:660666.

35 Brunelle JK, Bell EL, Quesada NM, et al. Oxygen sensing requires mitochondrial ROS but not oxidative phosphorylation. Cell Metab 2005; 1:409-414.

36 Guzy RD, Hoyos B, Robin E, et al. Mitochondrial complex III is required for hypoxia-induced ROS production and cellular oxygen sensing. Cell Metab 2005; 1:401-408.
37 An WG, Kanekal M, Simon MC, Maltepe E, Blagosklonny MV, Neckers LM. Stabilization of wild-type p53 by hypoxia-inducible factor 1alpha. Nature 1998; 392:405-408.

38 Graeber TG, Osmanian C, Jacks T, et al. Hypoxia-mediated selection of cells with diminished apoptotic potential in solid tumours. Nature 1996; 379:88-91.

39 Salceda S, Caro J. Hypoxia-inducible factor 1alpha (HIF-1alpha) protein is rapidly degraded by the ubiquitin-proteasome system under normoxic conditions. Its stabilization by hypoxia depends on redox-induced changes. J Biol Chem 1997; 272:22642-22647.

40 Huang LE, Gu J, Schau M, Bunn HF. Regulation of hypoxia-inducible factor 1alpha is mediated by an $\mathrm{O}_{2}$-dependent degradation domain via the ubiquitin-proteasome pathway. Proc Natl Acad Sci USA 1998; 95:7987-7992.

41 O'Rourke JF, Tian YM, Ratcliffe PJ, Pugh CW. Oxygen-regulated and transactivating domains in endothelial PAS protein 1: comparison with hypoxia-inducible factor-1alpha. $J$ Biol Chem 1999; 274:2060-2071.

42 Epstein AC, Gleadle JM, McNeill LA, et al. C. elegans EGL9 and mammalian homologs define a family of dioxygenases that regulate HIF by prolyl hydroxylation. Cell 2001; 107:4354.

43 Bruick RK, McKnight SL. A conserved family of prolyl-4-hydroxylases that modify HIF. Science 2001; 294:1337-1340.

44 Berra E, Benizri E, Ginouves A, Volmat V, Roux D, Pouyssegur J. HIF prolyl-hydroxylase 2 is the key oxygen sensor setting low steady-state levels of HIF-1alpha in normoxia. EMBO J 2003; 22:4082-4090.

45 Kaelin WG. Proline hydroxylation and gene expression. Ann Rev Biochem 2005; 74:115-128.

46 Schofield CJ, Ratcliffe PJ. Oxygen sensing by HIF hydroxylases. Nat Rev Mol Cell Biol 2004; 5:343-354.

47 Hagen T, Taylor CT, Lam F, Moncada S. Redistribution of intracellular oxygen in hypoxia by nitric oxide: effect on HIF1alpha. Science 2003; 302:1975-1978.

48 Ivan M, Haberberger T, Gervasi DC, et al. Biochemical purification and pharmacological inhibition of a mammalian prolyl hydroxylase acting on hypoxia-inducible factor. Proc Natl Acad Sci USA 2002; 99:13459-13464.

49 Sudarshan S, Sourbier C, Kong HS, et al. Fumarate hydratase deficiency in renal cancer induces glycolytic addiction and hypoxia-inducible transcription factor 1alpha stabilization by glucose-dependent generation of reactive oxygen species. Mol Cell Biol 2009; 29:4080-4090.

50 Kaelin WG Jr. The von Hippel-Lindau tumour suppressor protein: $\mathrm{O}_{2}$ sensing and cancer. Nat Rev Cancer 2008; 8:865873.

51 Ivan M, Kondo K, Yang H, et al. HIFalpha targeted for VHL-mediated destruction by proline hydroxylation: implications for $\mathrm{O}_{2}$ sensing. Science 2001; 292:464-468.

52 Jaakkola P, Mole DR, Tian YM, et al. Targeting of HIF-alpha to the von Hippel-Lindau ubiquitylation complex by $\mathrm{O}_{2}$-regulated prolyl hydroxylation. Science 2001; 292:468-472.

53 Maxwell PH, Wiesener MS, Chang GW, et al. The tumour suppressor protein VHL targets hypoxia-inducible factors for oxygen-dependent proteolysis. Nature 1999; 399:271-275.

54 Tanimoto K, Makino Y, Pereira T, Poellinger L. Mechanism of regulation of the hypoxia-inducible factor- 1 alpha by the 
von Hippel-Lindau tumor suppressor protein. EMBO J 2000; 19:4298-4309.

55 D'Angelo G, Duplan E, Boyer N, Vigne P, Frelin C. Hypoxia up-regulates prolyl hydroxylase activity: a feedback mechanism that limits HIF-1 responses during reoxygenation. J Biol Chem 2003; 278:38183-38187.

56 Marxsen JH, Stengel P, Doege K, et al. Hypoxia-inducible factor-1 (HIF-1) promotes its degradation by induction of HIF-alpha-prolyl-4-hydroxylases. Biochem J 2004; 381:761767.

57 del Peso L, Castellanos MC, Temes E, et al. The von Hippel Lindau/hypoxia-inducible factor (HIF) pathway regulates the transcription of the HIF-proline hydroxylase genes in response to low oxygen. J Biol Chem 2003; 278:48690-48695.

58 Foxler DE, Bridge KS, James V, et al. The LIMD1 protein bridges an association between the prolyl hydroxylases and VHL to repress HIF-1 activity. Nat Cell Biol 2012; 14:201208.

59 Sharp TV, Al-Attar A, Foxler DE, et al. The chromosome 3p21.3-encoded gene, LIMD1, is a critical tumor suppressor involved in human lung cancer development. Proc Natl Acad Sci USA 2008; 105:19932-19937.

60 Sharp TV, Munoz F, Bourboulia D, et al. LIM domains-containing protein 1 (LIMD1), a tumor suppressor encoded at chromosome $3 \mathrm{p} 21.3$, binds pRB and represses E2F-driven transcription. Proc Natl Acad Sci USA 2004; 101:1653116536.

61 Ferreira JV, Fofo H, Bejarano E, et al. STUB1/CHIP is required for HIF1A degradation by chaperone-mediated autophagy. Autophagy 2013; 9:1349-1366.

62 Olmos G, Arenas MI, Bienes R, et al. 15-Deoxy-Delta(12,14)-prostaglandin-J(2) reveals a new pVHL-independent, lysosomal-dependent mechanism of HIF-1alpha degradation. Cell Mol Life Sci 2009; 66:2167-2180.

63 Hubbi ME, Hu H, Kshitiz, Ahmed I, Levchenko A, Semenza GL. Chaperone-mediated autophagy targets hypoxia-inducible factor-1alpha (HIF-1alpha) for lysosomal degradation. $J$ Biol Chem 2013; 288:10703-10714.

64 Berthold J, Schenkova K, Rivero F. Rho GTPases of the RhoBTB subfamily and tumorigenesis. Acta Pharmacol Sin 2008; 29:285-295.

$65 \mathrm{Lu}$ A, Pfeffer SR. Golgi-associated RhoBTB3 targets cyclin E for ubiquitylation and promotes cell cycle progression. $J$ Cell Biol 2013; 203:233-250.

66 Schenkova K, Lutz J, Kopp M, Ramos S, Rivero F. MUF1/ leucine-rich repeat containing 41 (LRRC41), a substrate of RhoBTB-dependent cullin 3 ubiquitin ligase complexes, is a predominantly nuclear dimeric protein. $J$ Mol Biol 2012; 422:659-673.

67 Berthold J, Schenkova K, Ramos S, et al. Characterization of RhoBTB-dependent Cul3 ubiquitin ligase complexes - evidence for an autoregulatory mechanism. Exp Cell Res 2008; 314:3453-3465.
68 Espinosa EJ, Calero M, Sridevi K, Pfeffer SR. RhoBTB3: a Rho GTPase-family ATPase required for endosome to Golgi transport. Cell 2009; 137:938-948.

69 Koivunen P, Lee S, Duncan CG, et al. Transformation by the (R)-enantiomer of 2-hydroxyglutarate linked to EGLN activation. Nature 2012; 483:484-488.

70 Yu F, White SB, Zhao Q, Lee FS. HIF-1alpha binding to VHL is regulated by stimulus-sensitive proline hydroxylation. Proc Natl Acad Sci USA 2001; 98:9630-9635.

71 Isaacs JS, Jung YJ, Mimnaugh EG, Martinez A, Cuttitta F, Neckers LM. Hsp90 regulates a von Hippel Lindau-independent hypoxia-inducible factor-1 alpha-degradative pathway. $J$ Biol Chem 2002; 277:29936-29944.

72 Minet E, Mottet D, Michel G, et al. Hypoxia-induced activation of HIF-1: role of HIF-1alpha-Hsp90 interaction. FEBS Lett 1999; 460:251-256.

73 Song D, Li LS, Heaton-Johnson KJ, Arsenault PR, Master SR, Lee FS. Prolyl hydroxylase domain protein 2 (PHD2) binds a Pro-Xaa-Leu-Glu motif, linking it to the heat shock protein 90 pathway. J Biol Chem 2013; 288:9662-9674.

74 Yusenko MV, Kuiper RP, Boethe T, Ljungberg B, van Kessel AG, Kovacs G. High-resolution DNA copy number and gene expression analyses distinguish chromophobe renal cell carcinomas and renal oncocytomas. BMC Cancer 2009; 9:152.

75 Beroukhim R, Brunet JP, Di Napoli A, et al. Patterns of gene expression and copy-number alterations in von-hippel lindau disease-associated and sporadic clear cell carcinoma of the kidney. Cancer Res 2009; 69:4674-4681.

76 Gumz ML, Zou H, Kreinest PA, et al. Secreted frizzled-related protein 1 loss contributes to tumor phenotype of clear cell renal cell carcinoma. Clin Cancer Res 2007; 13:4740-4749.

77 Rui Y, Xu Z, Lin S, et al. Axin stimulates p53 functions by activation of HIPK2 kinase through multimeric complex formation. EMBO J 2004; 23:4583-4594.

78 Callapina M, Zhou J, Schmid T, Kohl R, Brune B. NO restores HIF-1alpha hydroxylation during hypoxia: role of reactive oxygen species. Free Radic Biol Med 2005; 39:925-936.

79 Patten DA, Lafleur VN, Robitaille GA, Chan DA, Giaccia AJ, Richard DE. Hypoxia-inducible factor-1 activation in nonhypoxic conditions: the essential role of mitochondrial-derived reactive oxygen species. Mol Biol Cell 2010; 21:3247-3257.

80 Liu W, Xin H, Eckert DT, Brown JA, Gnarra JR. Hypoxia and cell cycle regulation of the von Hippel-Lindau tumor suppressor. Oncogene 2011; 30:21-31.

(Supplementary information is linked to the online version of the paper on the Cell Research website.)

cc)(i) (-) $\Theta$ This work is licensed under the Creative Commons Attribution-NonCommercial-No Derivative Works 3.0 Unported License. To view a copy of this license, visit http:// creativecommons.org/licenses/by-nc-nd/3.0 